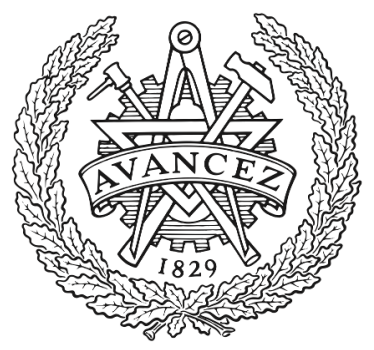

CHALMERS

UNIVERSITY OF TECHNOLOGY

\title{
Trends in vehicle motion control for automated driving on public roads
}

Downloaded from: https://research.chalmers.se, 2023-04-26 12:33 UTC

Citation for the original published paper (version of record):

Klomp, M., Jonasson, M., Laine, L. et al (2019). Trends in vehicle motion control for automated driving on public roads. Vehicle System Dynamics, 57(7): 1028-1061.

http://dx.doi.org/10.1080/00423114.2019.1610182

N.B. When citing this work, cite the original published paper. 


\title{
Trends in Vehicle Motion Control for Automated Driving on Public Roads
}

\author{
Matthijs Klomp ${ }^{\mathrm{a}}$, Mats Jonasson ${ }^{\mathrm{b}}$, Leo Laine ${ }^{\mathrm{c}}$, Leon Henderson ${ }^{\mathrm{c}}$, Enrico Regolin ${ }^{\mathrm{d}}$ \\ and Stefan Schumi ${ }^{\mathrm{e}}$ \\ a Volvo Car Group, Gothenburg, Sweden; ${ }^{\text {b }}$ Chalmers University of Technology, Gothenburg,

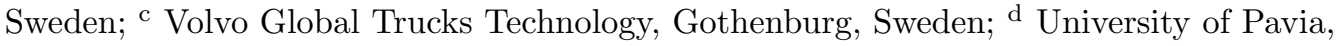 \\ Pavia, Italy; ${ }^{e}$ Infineon Technologies, Neubiberg, Germany
}

\section{ARTICLE HISTORY}

Compiled May 3, 2019

\begin{abstract}
In this paper we describe how vehicle systems and the vehicle motion control are affected by automated driving on public roads. We describe the redundancy needed for a road vehicle to meet certain safety goals. The concept of system safety as well as system solutions to fault tolerant actuation of steering and braking and the associated fault tolerant power supply is described. Notably restriction of the operational domain in case of reduced capability of the driving automation system is discussed. Further we consider path tracking, state estimation of vehicle motion control required for automated driving as well as an example of a minimum risk maneuver and redundant steering by means of differential braking. The steering by differential braking could offer heterogeneous or dissimilar redundancy that complements the redundancy of described fault tolerant steering systems for driving automation equipped vehicles. Finally the important topic of verification of driving automation systems is addressed.
\end{abstract}

\section{KEYWORDS}

chassis control systems; autonomous vehicles; fault-tolerant control; integrated control systems; state estimation; system safety

\section{Introduction}

Automated driving has attracted considerable interest from industry and the general public. The main drivers for automated driving are that connected automated vehicles have the potential to improve traffic safety, fuel efficiency and traffic flow 11. According to 2 automated vehicles may also result in less required parking and fewer vehicles, but more distance traveled in total. They point out that obstacles to adoption include cost, liability, licensing, security, and privacy concerns.

The main elements of manual or automated driving are perception, decision and control. In the context of driving automation systems, most commonly referenced is SAE J3016 [3], which describes driving automation systems that perform part or all of the so-called dynamic driving task on a sustained basis. The standard provides detailed definitions for six levels of driving automation, ranging from no driving automation 
(level 0) to full driving automation (level 5). The intention of this paper is to be consistent with the definitions of this standard.

Examples of level 1 automation systems include adaptive cruise control (longitudinal control only), which have seen very wide industry adoption. When cruise control is combined with lateral control, such as a lane centering system, this would constitute a level 2 automation system. In level 3 automation systems the entire dynamic driving task within a limited Operational Driving Domain (ODD) ${ }^{1}$ is performed by the automation system but requires the user to take over the driving task in case of a system failure. In a level 4 driving automation system, the fall-back operation will also be provided by the driving automation system in case the user is not available or until he/she is ready to take over the driving task. In a level 5 automation system, the difference to a level 4 automation system is that there are no restrictions on the operating envelope of the vehicle. In some cases no human driver is even present in the vehicle, such as such as the Vera and 360c concepts shown in Fig. 1 and which were recently presented by Volvo Trucks and Volvo Cars, respectively.

One way of achieving improved road safety, road utilization and decrease fuel consumption through driving automation is via the concept of cooperative driving [4] involving an intelligent infrastructure as well as vehicle to vehicle cooperation. By cooperative driving, information about the driving environment is shared, thus enabling the involved vehicles to make more intelligent choices about the trajectory that is followed. One common example of cooperative driving is platooning, where a lead vehicle tows a platoon of vehicles. Studies such as [5] 7$]$ indicate that fuel savings of about 10 to $20 \%$ are possible, depending on the gap between the vehicles and number of vehicles in the platoon. Communication between vehicles in a platoon is typically done via the IEEE $802.11 \mathrm{p}$ vehicle-to-vehicle radio channel [8].

Automated driving on public roads will require a redesign of the vehicle to meet new requirements on functional safety and fault tolerance. Other industries, such as nuclear power plants, aviation, elevators, robots 9 have long dealt with these requirements, but often without the cost pressure present in the automotive industry. Triple (or higher) redundancy is usually applied to allow for being tolerant to multiple faults. The Space Shuttle, for instance, carried five general purpose computers, four being fully-redundant and the fifth being used only for mission critical stages such as launch and landing [10]. The system was predicted to cause a loss of a Space Shuttle only four times in a billion flights [11]. Currently, at least single fault tolerant systems are being proposed for steering, braking and propulsion systems for driving automation without a backup-ready user (SAE Level 4 and 5), as was described already in 12. Combining these redundant systems could in some cases lead to higher redundancy [13], as will be discussed further in this paper.

A comprehensive review of fundamental goals, development and perspectives of driving automation systems can be found in [14. Their work describes how driving automation systems are developed in an evolutionary fashion from driver support systems to increasingly higher levels of automation. Barriers to increased driving automation are described to be not only technical but also legislative. Another essential review on driving automation is [15] which gives an comprehensive historical overview of the evolution of driving automation as well as describing the main architecture and essential elements of driving automation systems. One major challenge in driving automation, not covered by this paper, are the tactical functions of object and

${ }^{1}$ The ODD are the specific conditions under which a given driving automation system or feature thereof is designed to function 3 . 
(a)

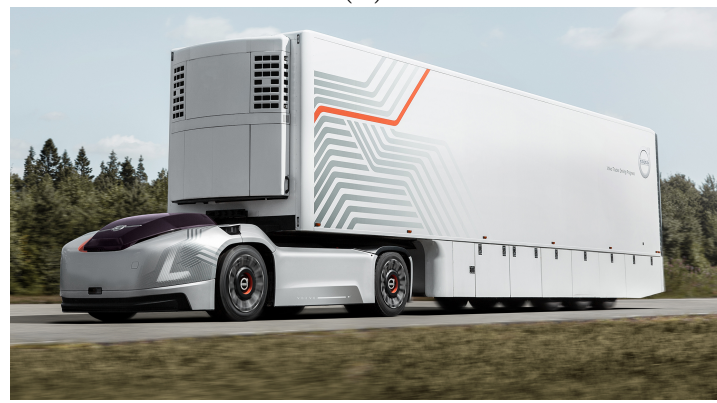

(b)

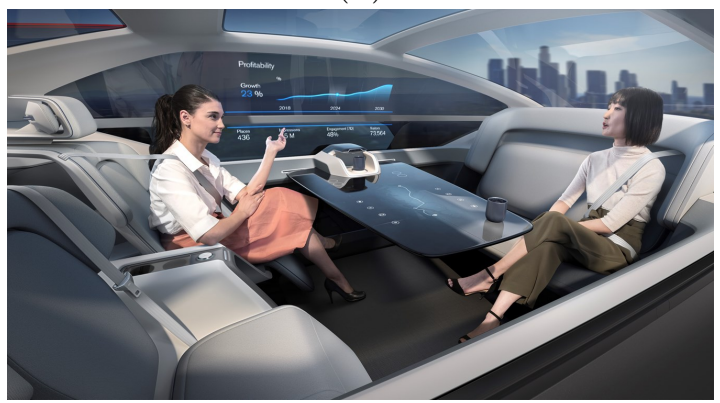

Figure 1.: Driverless concepts: Volvo Vera (a) and 360c Concept (b) (image credits: Volvo Trucks and Volvo Car Group, respectively)

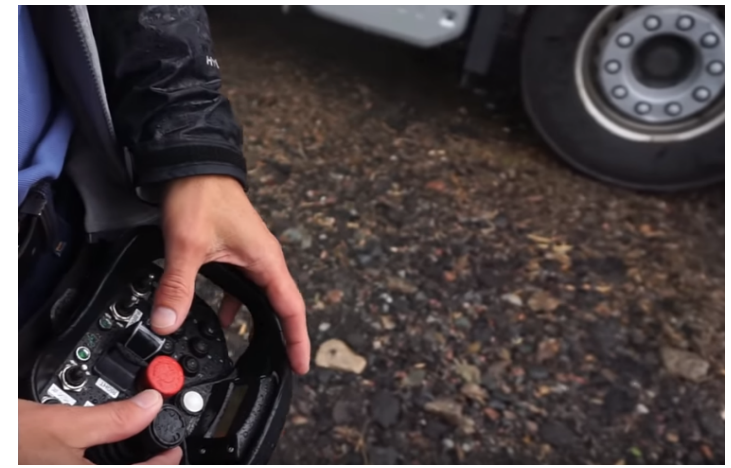

Figure 2.: Volvo external steering 22

event detection, classification and response which also includes path planning and replanning 16 18. For perception challenges we refer to a recent review on this topic in 19 .

The first introductions of automated vehicles on public roads will be in a mixed traffic environment with manual driving which increases the risks for hazards and hence the complexity of vehicle control increases. When the society has fully automated public road traffic, vehicle-to-X [20] will reveal objects round corners, which is not feasible for standard vehicle environment sensors such as LIDAR, camera etc of the subject vehicle. Backup driver controls are necessary in the case of a truck or car without driver controls (such as the Volvo 360c and Vera shown in Fig. 1) using frequency-hopping spread spectrum (FHSS) remote control, see Fig. 22 [21.

While the aforementioned papers provide a review of the overall challenges and state-of-the art of driving automation systems for fault tolerant operation 23, this present paper intends to focus on vehicle motion and chassis control $24-26]$ for driving automation systems.

The Vehicle Motion Control (VMC), which is the operative part of the driving automation system (see Fig. 3), needs a common reference for the desired vehicle motion in the road plane. The reference motion is coordinated among available motion devices by the VMC. The VMC also provides status and capabilities of feasible motion to the Automated Driving System (ADS). The integrated vehicle motion control is a necessity in order to assure that the vehicle can be safely operated in autonomous mode. In [27] a similar control structure was proposed and successfully applied to real 


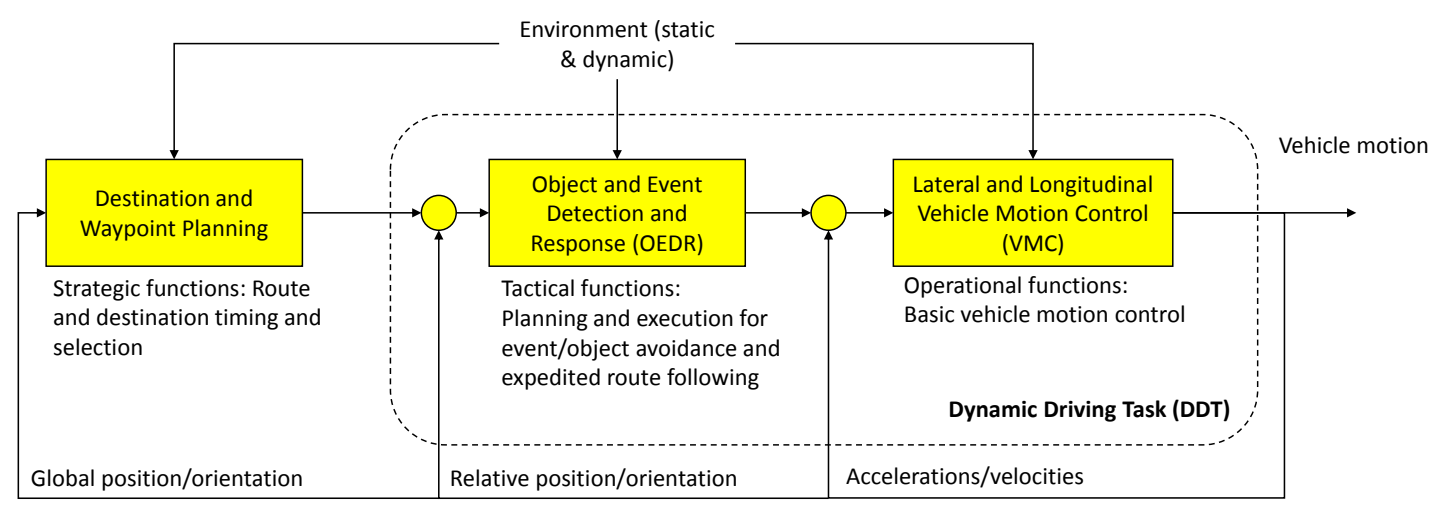

Figure 3.: Schematic view of the driving task (adapted from 3])

traffic situations. An comprehensive paper on functional architectures for automated driving can be found in [28] where the different parts shown in Fig. 3 are described in detail with examples from different published implementations.

In this paper we discuss how the vehicle control, actuation (primarily steering and braking) and power distribution will be affected by automated driving on public roads. In addition we will also describe the redundancy needed for fault tolerant operation, for a road vehicle to meet certain system safety requirements.

\section{Automated driving on public roads}

In this paper we will focus on standard maneuvering, by which we mean an ODD with roughly a lateral acceleration below $3 \mathrm{~m} / \mathrm{s}^{2}$ for cars and below $2 \mathrm{~m} / \mathrm{s}^{2}$ for trucks and braking below $5 \mathrm{~m} / \mathrm{s}^{2}$ for a car and $3.5 \mathrm{~m} / \mathrm{s}^{2}$ for a truck.

The first introductions of automated vehicles will be in mixed traffic environments with manual driving. This increases the risks for hazards and hence also increases the complexity of the vehicle control task.

In order to illustrate some of the basic challenges involved in developing a safe driving automation system (shown in Fig. 5), we will here show a very simple use case, see Fig. 4, where the ADS equipped vehicle is driving on a straight road with a vehicle ahead. The distance $L$ to the vehicle ahead is dependent on the road friction and the braking capability of the motion support brake devices. If it suddenly starts to rain, it is important that the vehicle environment sensors can detect the free space and vehicle ahead with high confidence during the rainy event. It is as important to have high confidence from the motion support device of brake torque capability and actual road friction estimate, which VMC can use to estimate the acceleration capability for braking with high confidence. Only then can the Object and Event Detection and Response (OEDR) management decide what distance $L$ is needed in the upcoming situation to be able to guarantee safe automated driving.

Looking only at braking, we have, with $a$ being the braking deceleration magnitude, that the stopping distance, $d$, obviously is

$$
d=\frac{1}{2} \frac{v^{2}}{a}
$$




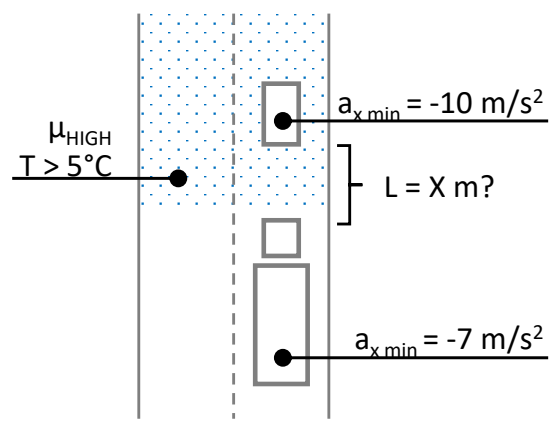

Figure 4.: Every day use case - driving on a straight road with sudden rain ahead with the challenge to decide distance $L$ to vehicle ahead.

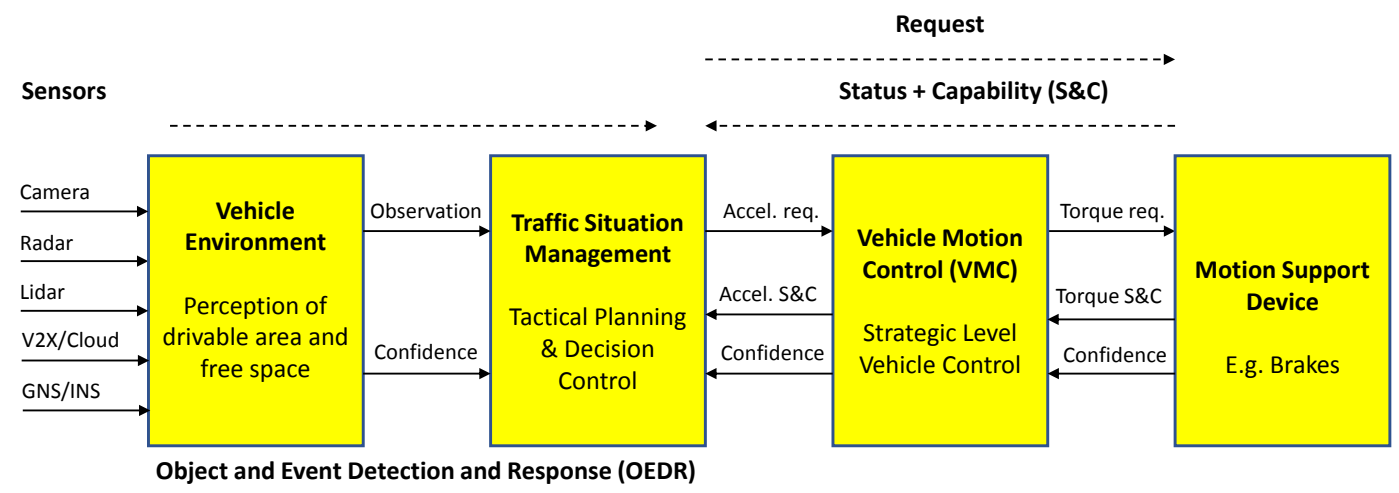

Figure 5.: Sensor Device

with $v$, being the vehicle speed. The stopping distance as function of speed is shown in Fig. 6. Given that we must be able to stop from (1), we then see that for $a=5 \mathrm{~m} / \mathrm{s}^{2}$, we thus have that our sensors must be able to detect a free-space of $90 \mathrm{~m}$ if we are to be able to stop within that distance. If instead we are only interested in being able to stop for a hard braking leading vehicle as discussed above, we can add the distance required for this vehicle to brake as hard as typically possible for a passenger vehicle to come to the safe distance $L$. If the sensor performance is degraded, for any reason, the speed must therefore be adapted to the available free space.

Although the above limitations probably will apply for a considerable time, this hasn't stopped inventive researchers to explore more extreme maneuvering. One such example is for instance [29 which focuses on non-linear path tracking following clothoid paths using combined braking/steering. In 30 fuzzy logic is applied to yaw moment stability control, which the authors claim is a simple method to achieve good performance in controlling non-linear systems. Even more advanced maneuvers are explored in, for instance, 31 where robust power slides are analyzed. More work on such high side-slip maneuvers can be found in 32 using a finite state machine approach, and in [33 using a hybrid hierarchical driver model and finally in [34] employing neural networks to perform these maneuvers. In [35] as well as 36 interesting analysis using phase portraits are employed to visualize the effects of controlling the highly non-linear regimes of the vehicle operating region. In 35 the evolution of system state at the different steer frequencies using bifurcation analysis of system state transformations are described in an interesting manner. Further analysis of accelerating and braking 


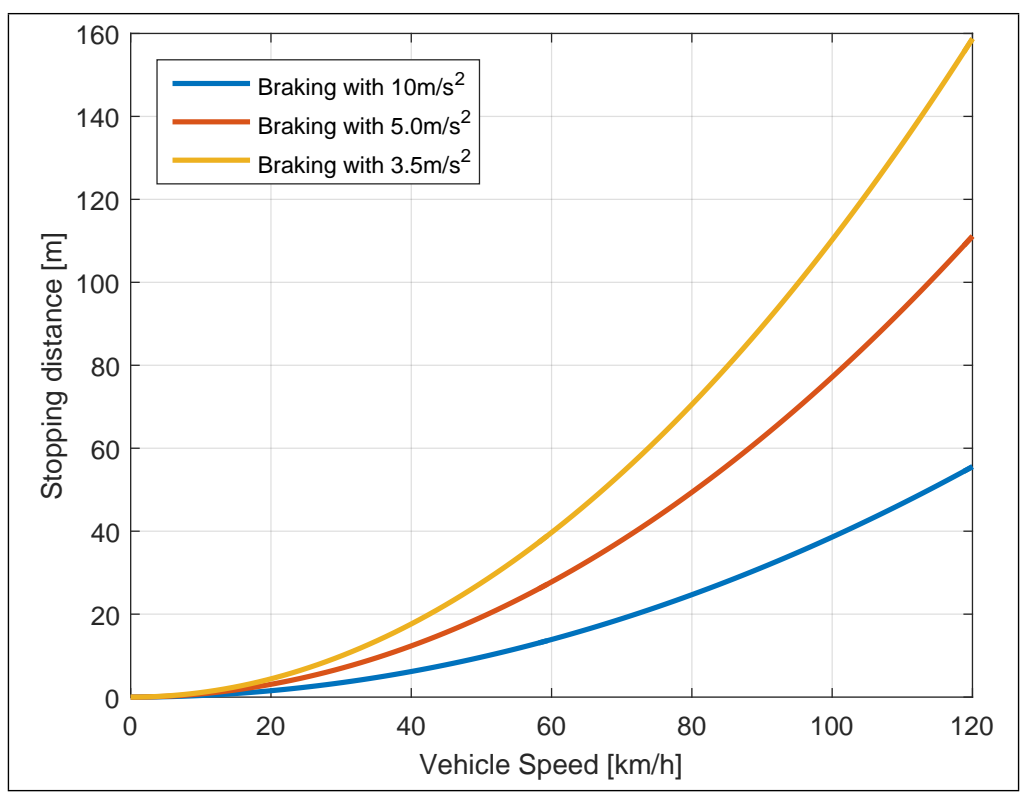

Figure 6.: Stopping distance vs speed for three different levels of deceleration.

stability using constrained bifurcation and continuation methods can be found in [37]. Another non-linear tracking method is a stable tracking with the front tires of the vehicle being saturated typically occurring during overspeeding in a turn. Work on recovering from such situations was developed in 38 and more recently in [39]. In [40] control allocation for avoidance maneuvers with oncoming vehicles is developed. There it is shown that for some combinations of obstacle length, ego vehicle and oncoming vehicle speed even acceleration may be required to minimize the collision risk. Similar results were obtained for intersection accidents in [41. One important aspect of the automated driving is to understand the vehicle limitations. One very interesting method to derive these limitations off-line was presented in [42] where an innovative method for the computation of the so-called controllability region is presented for different actuator combinations. Their method is formulated as an optimal control problem that maximize the norm of the initial condition of the vehicle yaw-rate and side-slip states, i.e. how far from the stationary condition the vehicle can be stabilized with the given set of actuators and road conditions. These limitations can then be applied with tolerance bounds on the ODD for different actuator combinations and road conditions.

In quasi-steady state conditions a method is proposed in 43 on how to find the optimal tire force distribution that maximizes the global vehicle force in a particular direction, which could be very useful in critical situations.

\section{System Safety and Fault-tolerant Actuation}

As mentioned in the introduction, the ADS equipped vehicle must be capable of handle faults in the control architecture. Faults in the vehicle motion control architecture could generate hazards and should be protected against.

To achieve system safety for advanced driving assistance systems (ADAS) several things have to be taken into consideration. Accounted has to be: Which function does 
the system provide? What can happen at failure? What is the safety mechanism at failure - Is it electrical, mechanical or can it be a human person? Who is responsible? An excellent survey on fault-tolerant vehicle design can be found in 44. They highlight that driveline electrification and new chassis systems enables higher levels of redundancy but also increase the number of fault possibilities. They conclude that a holistic view has to be considered to get a harmonized level of fault-tolerance. These aspects will be further discussed in this section as well as how they relate to system safety aspects within ISO 26262 and its application to driving automation systems and their development.

As described in the introduction, SAE J3016 [3] describes six levels of automation. As an example, an Electric Power Assisted Steering (EPAS) unit will be given. At Level 0 , there are only power assist functions available. According to the ISO 26262 [45], if this system does fail, it must reach a fail-safe state. In the rare case of failure the electric motor must not block (fail-silent) and the human driver may have to steer without any power assist. At level 1, the EPAS might warn the human driver if he/she is going out of lane and intervenes by slightly steering back into the lane (Lane Keeping Assist, LKA). Other level 1 automation functions are steering wheel torque overlay to mitigate oversteer or understeer [46]. At level 2, the EPAS can be used to continuously stay within the lane [47,48], but will require the driver to keep at all times his hands on the steering wheel and monitor the system. To ensure the driver is alert and ready to intervene at any time, hands-off detection (for instance [49]) and/or vision based driver monitoring systems [50] are employed. At level 3 the EPAS can perform the entire dynamic driving task. Thereby, the human driver might do other tasks. Systems safety is ensured by an alert to the human, who has to be ready to act immediately if a request to intervene (RTI) has been sent out. This RTI could be issued if there is any failure within the vehicle regarding the OEDR management or within the lateral or longitudinal VMC capability. From this point onward, any Level 3 functions of the vehicle are set back to Level 1 and the human driver is fully responsible. For braking, examples Level 1 automation are electronic stability control, which intervenes to stabilize the vehicle in case of a skid [51] or adaptive cruise control (ACC) described in the introduction and which automatically adjusts the vehicle speed to maintain a safe distance from vehicles ahead. Level 2 and higher automation is when ACC is combined with steering, as described above.

\subsection{System Safety}

Many hazards in automotive systems [52] are of the highest automotive safety integrity level: ASIL-D. This means that they can occur in common situations (exposure), the outcome is potentially life-threatening (severity) and difficult to control by the driver if they occur (see Tab. 1). Examples of such events and their ASIL-rating are shown in Tab. 2. System safety is about designing the system such that it is free from unreasonable risk caused by systematic faults or random faults by having an ASIL capability of the item (such as the brake system) that at least equal to the ASIL classification of the hazard (see Table 2] [45]). This is achieved by [53]:

(1) Performing a hazard \& risk analysis:

- Which loss of functionality may lead to a hazardous event ${ }^{2}$

(2) Defining safety goals:

- Functionality needed to avoid an unreasonable risk for a hazardous event

${ }^{2}$ Hazards caused by the functionality itself are covered in the safety of the intended functionality (SOTIF) 54. 


\begin{tabular}{|c|c|c|c|c|}
\hline Severity & Exposure & \multicolumn{3}{|c|}{ Controllability } \\
\hline \multirow{2}{*}{$\begin{array}{c}\text { Extent of harm to individual(s) } \\
\text { that can occur in hazardous } \\
\text { situation }\end{array}$} & \multirow{2}{*}{$\begin{array}{c}\text { Probability of exposure } \\
\text { regarding operational } \\
\text { conditions }\end{array}$} & \multicolumn{3}{|c|}{$\begin{array}{c}\text { Ability to avoid a specified harm through } \\
\text { timely reactions }\end{array}$} \\
\hline & & C1 - Simple & C2 - Normal & C3 - Difficult \\
\hline \multirow{4}{*}{$\begin{array}{l}\text { S1 - Light and moderate } \\
\text { injuries }\end{array}$} & E1 - Very low & $\mathrm{QM}$ & $\mathrm{QM}$ & $\mathrm{QM}$ \\
\hline & E2 - Low & $\mathrm{QM}$ & $\mathrm{QM}$ & $\mathrm{QM}$ \\
\hline & E3 - Medium & $\mathrm{QM}$ & $\mathrm{QM}$ & $\mathrm{A}$ \\
\hline & E4 - High & $\mathrm{QM}$ & $\mathrm{A}$ & $\mathrm{B}$ \\
\hline \multirow{4}{*}{$\begin{array}{c}\text { S2 - Severe and life- } \\
\text { threatening injuries (survival } \\
\text { probable) }\end{array}$} & E1 - Very low & $\mathrm{QM}$ & $\mathrm{QM}$ & QM \\
\hline & E2 - Low & QM & QM & $\mathrm{A}$ \\
\hline & E3 - Medium & $\mathrm{QM}$ & $\mathrm{A}$ & $\mathrm{B}$ \\
\hline & E4 - High & $\mathrm{A}$ & $\mathrm{B}$ & $\mathrm{C}$ \\
\hline \multirow{4}{*}{$\begin{array}{c}\text { S2 - Life-threatening injuries } \\
\text { (survival uncertain), fatal } \\
\text { injuries }\end{array}$} & E1 - Very low & $\mathrm{QM}$ & QM & $\mathrm{A}$ \\
\hline & E2 - Low & $\mathrm{QM}$ & $\mathrm{A}$ & $\mathrm{B}$ \\
\hline & E3 - Medium & $\mathrm{A}$ & $\mathrm{B}$ & $\bar{C}$ \\
\hline & E4 - High & $\mathrm{B}$ & $\mathrm{C}$ & $\mathrm{D}$ \\
\hline
\end{tabular}

Table 1.: Automotive Safety Integrity Levels (ASIL) according to 45

\begin{tabular}{|l|l|l|c|c|}
\hline Function & Class & Worst case environment/failure & Evaluation & ASIL \\
\hline \multirow{5}{*}{ Propulsion } & Omission & Railroad crossing / No acceleration & S3,E1,C2 & QM \\
& Omission & Highway / No acceleration & S1,E4,C1 & QM \\
& Commission & City (traffic light) / Sudden accel. & S3,E4,C3 & D \\
& Stuck at value & City / Constant acceleration & S3,E4,C3 & D \\
\hline \multirow{3}{*}{ Braking } & Omission & City / Loss of brake & S3,E4,C3 & D \\
& Commission & Highway / Sudden brake & S3,E4,C3 & D \\
& Stuck at value & Highway / Constant retardation & S2,E4,C2 & B \\
\hline \multirow{3}{*}{ Steering } & Omission & Highway / Loss of steering & S3,E4,C3 & D \\
& Commission & Highway / Sudden steering angle & S3,E4,C3 & D \\
& Stuck at value & Highway / Constant steering angle & S3,E4,C3 & D \\
\hline
\end{tabular}

Table 2.: Examples of hazards and corresponding ASIL classification from [55]

- Error prediction / detection

- Specify the safe state

(3) Developing safety concepts:

- Fault prevention: Probability of the fault manifesting is sufficiently low.

- Fault tolerance: Provide required functionality even in the presence of one or more faults.

- Fault prediction / detection: Health monitoring and start-up tests.

Below is a timing example from 53] from fault occurrence to reaching a safe state:

- $t_{1}$ : Fault with the potential to violate the safety goal occurs

- $t_{2}$ : Fault is detected

- $t_{3}$ : Beginning of emergency operation - execution of the warning and degradation strategy. Hazard is mitigated.

- $t_{4}$ : If the fault has not been mitigated until $t_{4}$ a hazardous event might occur.

- $t_{5}$ : System reaches a safe state (e.g. restricted vehicle state or standstill in a safe 


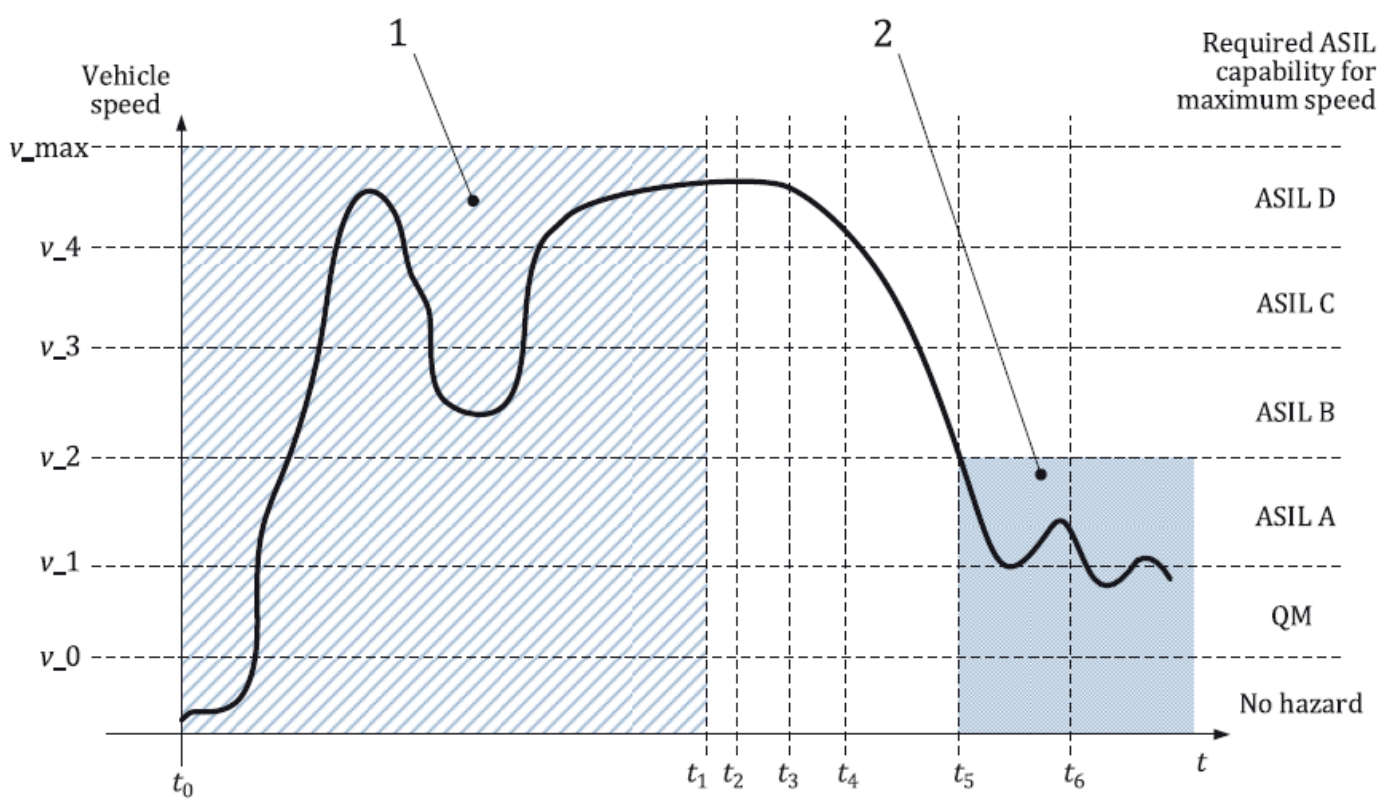

Figure 7.: Example of vehicle speed history according to Strategy 2. to restrict the ODD in the event of a failure from [45]. In area 1, the item is in normal operating mode with ASIL-D capability and in area 2, the item is in fault mode with ASIL-A capabiity.

location) or minimum risk condition ${ }^{3}$ End of emergency operation.

The safety concept shall ensure that $t_{3}-t_{1}<t_{4}-t_{1}$.

It should be noted that according to ISO 26262 :

During the emergency operation, the item is still free from unreasonable risk even though the ASIL capability of the item is lower than the ASIL rating of the possible hazard. To address this situation, the operating time in this state is limited, such that it is unlikely that an additional fault occurs which leads to a violation of the safety goal.

The duration of the emergency operation is defined and verified from the probability of a next fault.

According to [45], essentially two options exist once a safe state has been reached.

(1) The ADS is deactivated.

(2) The vehicle may continue to operate (without time restrictions) if the ODD is restricted such that the ASIL of the ODD does not exceed that of the remaining ASIL capability of the item.

In order to achieve the second option, fault operation strategies must be developed that restricts the ODD to comply with the above.

Another topic which is important for driving automation systems is the safety of the intended functionality (SOTIF), which is defined as absence of unreasonable risk due to hazards resulting from functional insufficiency of the intended functionality or by reasonably foreseeable misuse is now being standardized under ISO/PAS 21448 [54].

\footnotetext{
${ }^{3}$ According to 3 , a minimum risk condition is a condition to which a user or an ADS may bring a vehicle after performing the DDT fallback in order to reduce the risk of a crash when a given trip cannot or should not be completed.
} 
SOTIF implies robustness of the intended functionality (in our case, the driving automation capability) to a reasonable wide range of operating conditions. An excellent paper on robustness analysis of vehicle dynamics stability can can be found in 56 where they characterize the system robustness by evaluating the probabilities of violation of prescribed performance indexes through variation of uncertain vehicle parameters. They conclude that the employed stochastic approach improves the robustness of the evaluated vehicle control systems. Their work was followed up in [57] and [58 to aid the decision making process of the controller parameters applied to four-wheel steering and four-wheel torque control using a nonlinear predictive control again with the aim to improve the performance robustness to vehicle parameter variations. These methods could be further applied to the development of robust controllers for driving automation systems. Drive-by-Wire has already been in discussion for quite some time especially regarding the electronic driver assistance systems. Considerations towards structures with and without mechanical backup were discussed and concepts shown in [59].

\subsection{Fault-tolerant Braking and Steering Systems}

At Level 4 and 5 the human driver is allowed to do other tasks or might not even be present, which means that there might not be any response to the RTI in case of malfunction. If the system is fails without the user taking over the driving task, a fallback strategy is required such that a safe state can be reached. An EPS at this level of automation has to be fault-tolerant to achieve the required failure in time rating (FIT). As for steering, levels 4 and 5 require backup brake actuation in the rare case of complete failure of the primary brake actuation system.

For brake and steering systems there are usually two kinds of redundancy: diverse or homogeneous. Diverse redundancy is the use of multiple components of different types (e.g. use of different brake systems for the primary and backup system). Diverse (or heterogeneous) redundancy is more resistant to systematic faults, that is faults that are caused by design or manufacturing flaws 60 . In homogeneous redundancy, however multiple elements of a single type (e.g., use of dual, but identical electronic units and power stages for the steering actuation motor) are used to achieve redundancy. Homogeneous redundancy is more simple to implement and maintain but is susceptible to systematic faults. Both types of redundancies provide tolerance to random faults.

For steering several redundancy options are available. Examples include either two separate electric motors, a single induction motor with two independent three-phase circuits [61,62 or a single three-phase motor [63]; all powered by two independent power stages. Furthermore, both power stages need independent micro-controllers with independent power supply and communication.

\subsection{Fault-tolerant Electric Power Distribution}

Because automation systems are electric systems, the electric power distribution has to undergo significant changes too. They inherit the functional safety integration level of the defined function in automated mode. The current tree-shaped power distribution with a single lead-acid battery and an alternator do not meet the requirements 65 , 66 . In this section the development from current electric power supply through $48 \mathrm{~V}$ systems in mild hybrids to $400 \mathrm{~V}$ and higher in plugin-hybrids and battery electric vehicles, are discussed. 


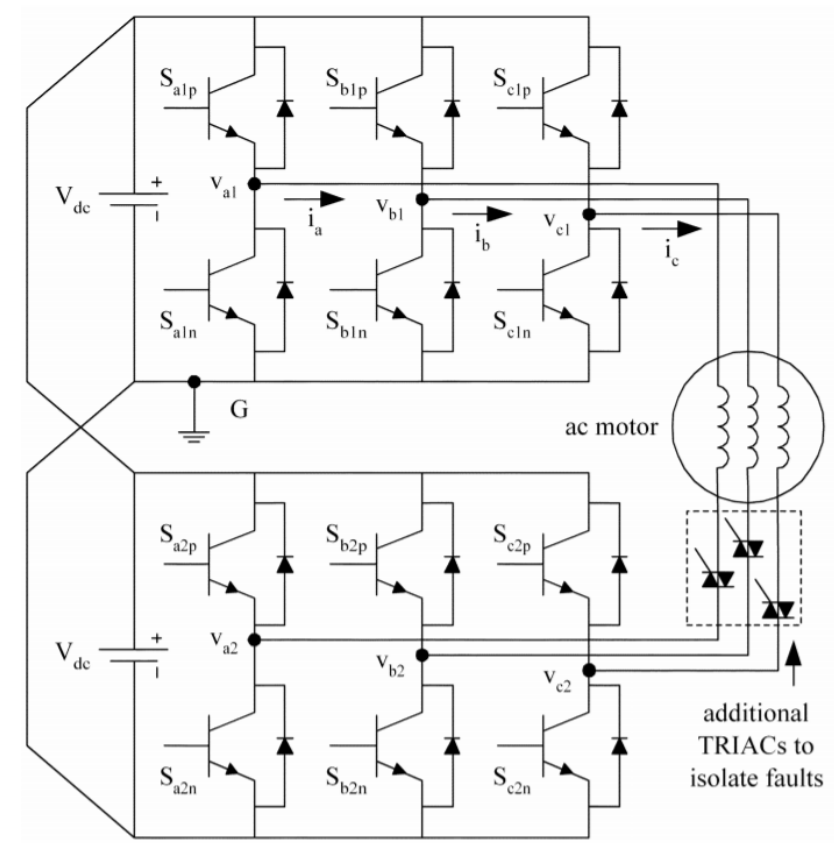

Figure 8.: Fault tolerant three phase motor with dual power stages 63.

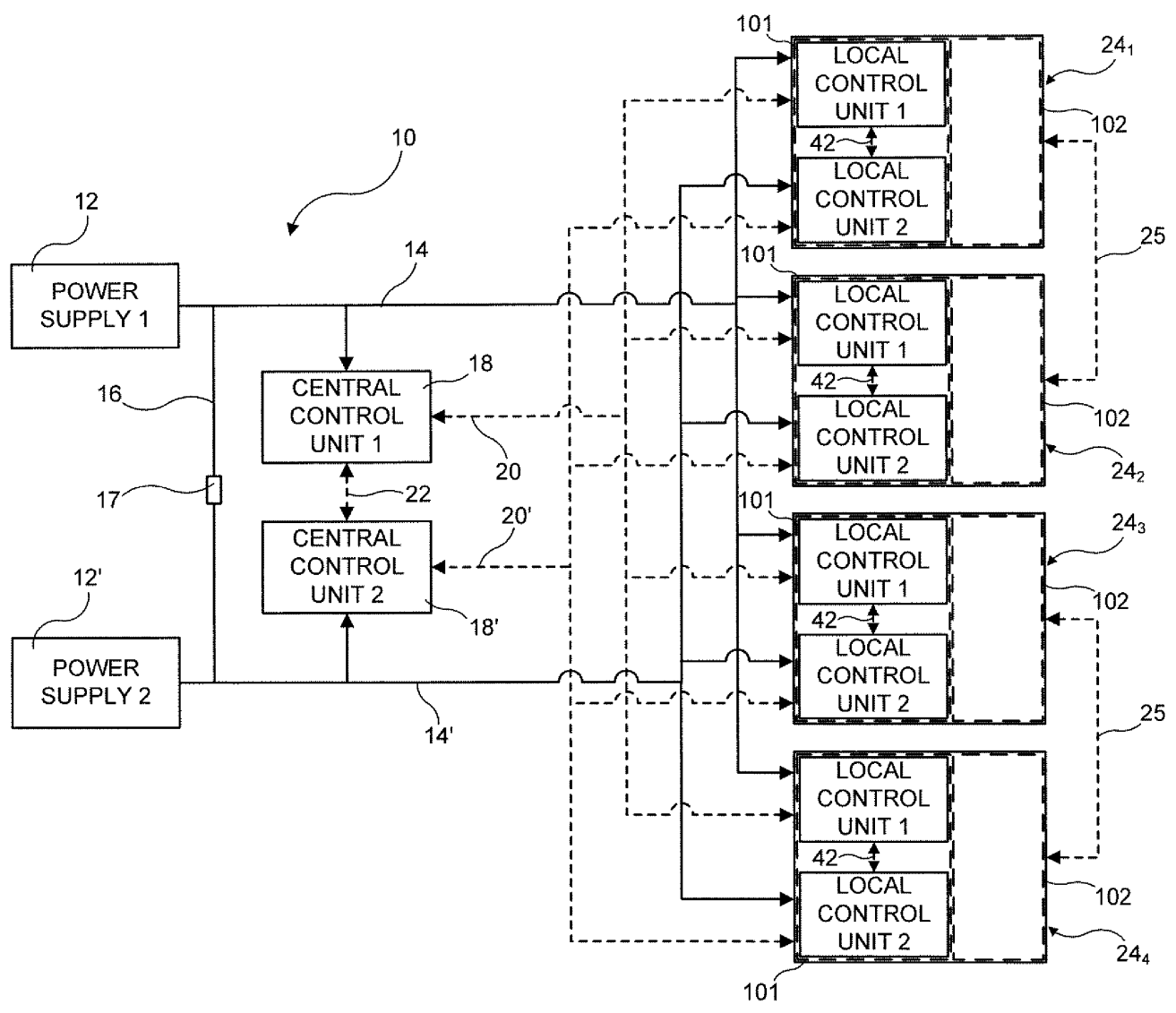

Figure 9.: Redundant brake actuators for fail safe brake system for heavy vehicles 64$]$. 


\subsubsection{Combustion and mild hybrid vehicles}

Combustion and mild hybrid vehicles have an alternator in the system. Combustion only vehicles, powered by $12 \mathrm{~V}$ only, look to loose market share in time, because of emission regulations all over the world and the rapid development of battery technology. Furthermore, the increase of applications for safety and consumer electronics are driving the need for higher and efficient electric power generation. This is enabled with the $48 \mathrm{~V}$ technology without the high isolation and safety requirements of high voltage (HV) systems 67 69. The electric power generation in both cases is done through an alternator connected with to the combustion engine [70]. $12 \mathrm{~V}$ systems are always powered through belt driven generators with a maximum power output of up to $6 \mathrm{~kW}[71] .48 \mathrm{~V}$ alternators, on the other hand, can also be placed as a flywheel integrated alternator with an output power of up to $15 \mathrm{~kW}$. The batteries in the system are usually charged, as long as the combustion engine is running. In case of a fault in the alternator, the control of the alternator or the rectifying part, the battery is the only remaining source to continue to supply the system. Depending on the attached loads, a $12 \mathrm{~V}$ battery can be drained within 20 minutes. This is in many cases not acceptable for L4 and L5 automated driving.

\subsubsection{High voltage hybrid vehicles and battery electric vehicles}

High voltage hybrids are similar in their setup to previous architectures. The only difference is that the alternator and the battery are operating at even higher voltage. In high voltage hybrid electric vehicles as well as in Battery Electric Vehicles (BEVs), an isolated DC/DC converter, instead of a $12 \mathrm{~V}$ alternator, is used to power to the $12 \mathrm{~V}$ system to which safety critical loads such as steering and braking as well critical sensing, decision and control functions are connected. The $12 \mathrm{~V}$ battery is then used in case of a fault in the DC/DC converter. Generation of electric energy during driving is only done through recuperation.

\subsubsection{Fail-Operational Concepts for Automated Driving}

All described architectures for mild-hybrids, high-voltage hybrids and BEVs are only fail-safe architectures. If the system is failing it is being switched off. Because the driver has to be present at all times and mechanical connections are available, the hazards can be avoided. With Level 3 and higher driving automation systems, both the communication 7275 and electric power distribution has to be fault-tolerant. A failure in the electric system should not lead to a hazard, it has to be avoided, as stated in 45$]$.

An ASIL-D hazard must be minimized to a target of $10 F I T=1 \cdot 10^{-8} h^{-1}$ as maximum limit for such critical functions [76]. Part of the power distribution for automated driving is the function to supply the safety relevant loads, which now is required to fulfill much higher availability than previously, thereby increasing the overall complexity of the system. The fail-operational concepts have to cover and supply the controller for the trajectory planning, sensor fusion, braking, steering and all other automated driving relevant loads. This means they have to be supplied independently from two power supply paths, as described in for instance [77]. Due to recharging possibilities those will have a connection but this connection must be able to be disconnected to achieve independence from each other, otherwise the concepts would not work. Two energy buffers are required for the system, because automated driving requires continuously more than $2 \mathrm{~kW}$ of electric power. A voltage drop due to high inrush currents 


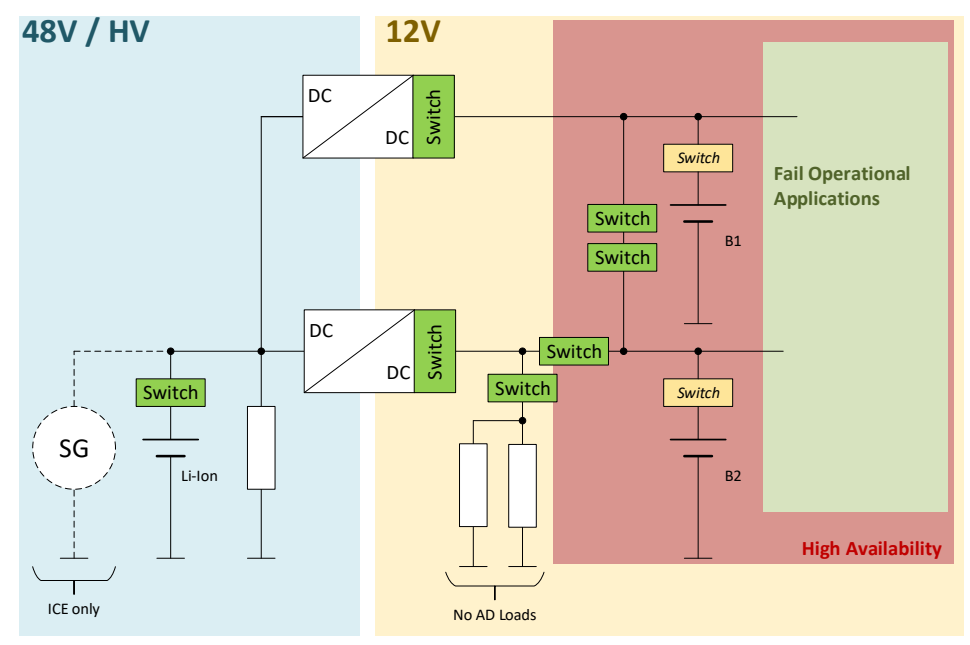

Figure 10.: L4/L5 dual DC/DC converter supply concept

should be avoided.

The operating voltage of current fail-operational concepts is $12 \mathrm{~V}$. Combining different voltage levels could be possible. HV power supply will likely never be used for safety critical applications and $48 \mathrm{~V}$ has some obstacles to replace $12 \mathrm{~V}$. Safety mechanisms and precautions must be taken to overcome them. Currently, it cannot be used as a backup/redundant supply if the safety precautions are not included. This increases the complexity and the costs of the system. A fail-operational concept for a highly automated vehicle can be seen in figure 10. This concept is powered through two $\mathrm{DC} / \mathrm{DC}$ converters and the bridge between the $12 \mathrm{~V}$ distribution. Latter one enables the charging of the second battery if one DC/DC converter fails. Failures in any of the $\mathrm{DC} / \mathrm{DC}$ converter resulting in a disconnection from the power distribution are happening more often than the requirement for the power distribution in automated vehicles allows. Further different concepts have been discussed and analyzed in 78 and [79]. Even this system does not meet the requirements fully yet. Also, the FIT rate for the batteries and DC/DC converters need to be lowered.

\subsubsection{Distribution for Automated Driving}

Considering the current tree shaped power distribution, all wires, supplies and loads are protected with standard melting fuses. For the high current loads those fuses are usually not to be exchanged by the end customer. These large fuses can be found usually close to the main battery and in the pre-fusebox (figure 11a) and are undergoing a change towards semiconductor fuses as shown in the right side of the figure.

Automated driving functions rely on a safe and stable power supply. Melting fuses are therefore not applicable for all applications. Not every short circuit is a low-ohmic short circuit at which a melting fuse might not blow. A safer and fault tolerant solution has to be implemented, which can act quickly and provide a safe (dis-)connection for the relevant functions. Electronics will thus replace the majority and safety critical fuses.

Figure $11 \mathrm{~b}$ shows the first of such an implementation, which is the power distribution center (PDC) of the Tesla Model 3 and which has some drawbacks. A single fault can be mitigated but a latent fault can lead to a hazard. The electronic fuse concept 


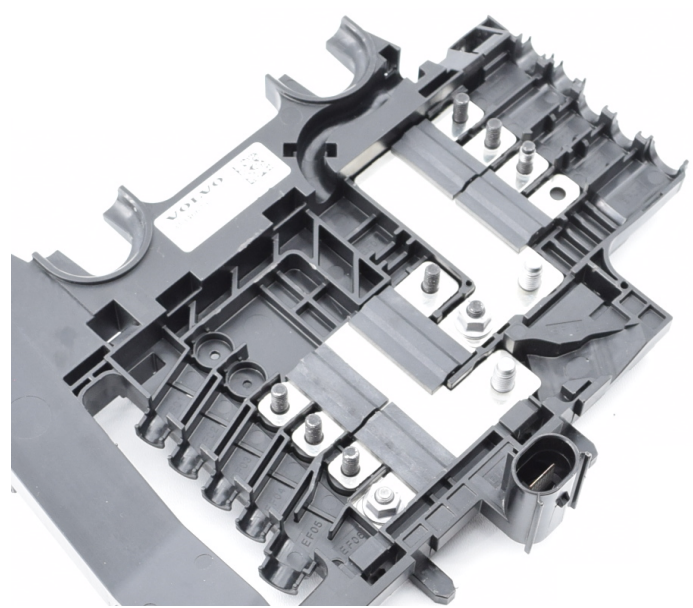

(a) Fusebox of Volvo S90

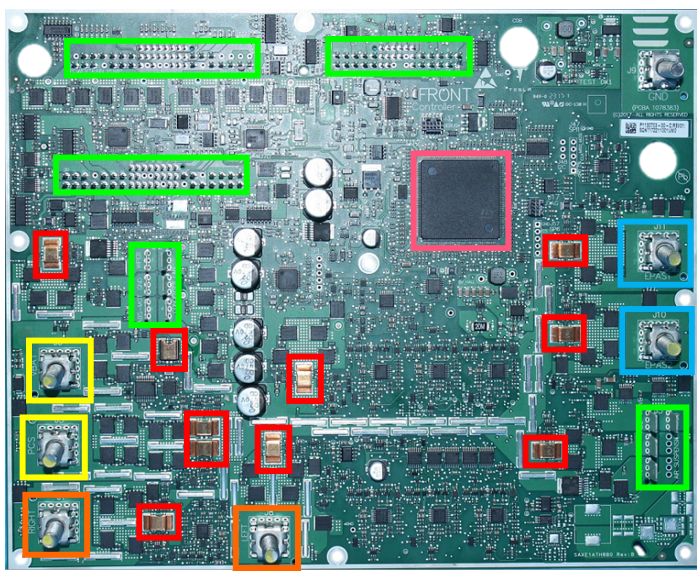

(b) PDC of Tesla Model 3

Figure 11.: Power distribution and fusing. Source: A2mac1

can be seen by the copper colored shunt resistors (red) in combination with the metaloxide-semiconductor field-effect transistors (MOSFET) close by. The entry-level power supply (blue) does feature two independent connections. Many other loads such as the braking and low power loads are connected to one of the other five connections (green). The system is supplied through either the battery or the DC/DC converter (yellow). There are two other PDCs in the Tesla Model 3 which are connected at the lower left side (orange). At those PDCs other single supplied loads are connected. Last but not least, there is a micro-controller (pink) on the board, coordinating the measurements and the actions of the board. Due to safety considerations this micro-controller has to be a safety controller. This system is not perfect yet but for L2 sufficient, as in the Model 3. A single point failure with a latent fault can still lead to a full loss of service of the board. Moving to higher automation, additional elements are thus required. Loads such as braking, sensor fusion and path planning do require a fuse of their own. Sensors can be coupled to a bigger fuse, if sufficient degree of freedom for the control of the vehicle is provided. Non driving relevant loads, can be merged to one (melting) fuse to save costs, similar as the orange paths.

\section{Vehicle motion function reference architecture}

The way logical/functional systems are divided into sub-systems are mentioned to as reference function architecture. Function reference architectures for vehicle motion are described in 80, 81.

One example of a typical vehicle motion control architecture for autonomous vehicles is seen in Fig. 12. The architecture is divided into three streams; longitudinal control, lateral control and vehicle state. This is done since these steams can act relatively independently from each other. The architecture is designed in a way that the AD-function could request a small set of high-level motion requests, which here is acceleration and path requests. The reason for requesting high level entities is that the AD-function should be relieved from the responsibility of having inherent and detailed knowledge of the actuators and vehicle dynamics sensors. Another reason is the possibility to isolate algorithms with different requirements on system safety lev- 


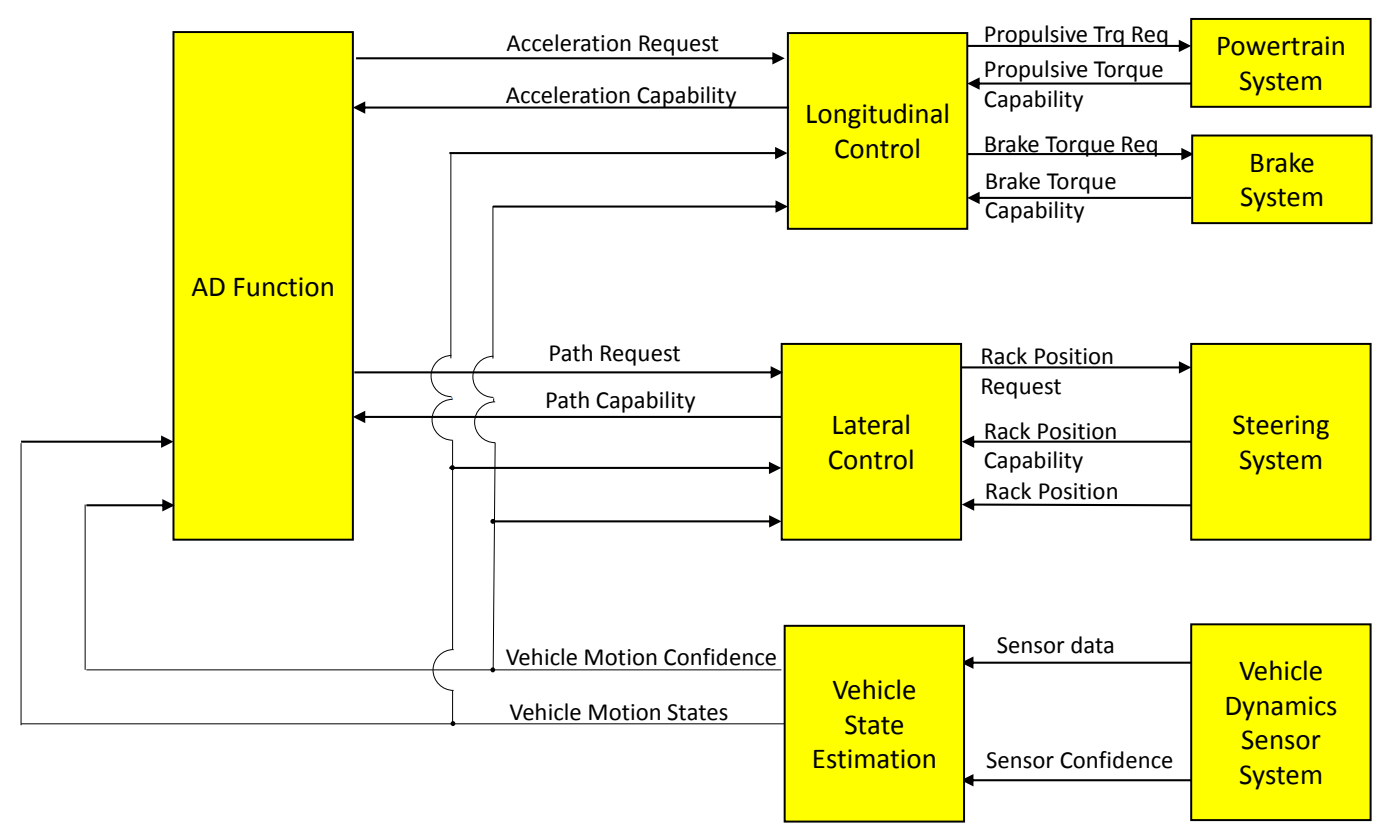

Figure 12.: Overview of vehicle motion control architecture suitable for automated driving vehicles

els. Information based on capabilities from the longitudinal and lateral controllers and confidence of vehicle state forms the constraints for the AD-function. The AD-function outputs an acceleration request to the longitudinal controller and a path request to the lateral controller. Less confidence of vehicle motion states and low capabilities from longitudinal and lateral control force the AD-function to be more conservative e.g. by lowering the vehicle speed. The vehicle dynamics sensor system typically consists of wheel speed sensors, steering angle sensors, inertial measurement units, sensors measuring states and status of the actuators.

\subsection{Path tracking}

The path tracking problem is an issue that concerns many different areas of research, from robotic manipulators to vessels. Therefore, although in the literature numerous studies can be found, a comprehensive analysis of all the proposed path tracking methods is very difficult 82 . In particular, the definition of state-of-the-art might vary according to the specific application considered. In recent years, Paden et al. 83 focused on producing a survey of the state of the art on planning and control algorithms for road vehicles, with a special focus on the urban setting. In there an overview is given at what are currently the standard control techniques in Motion Planning, i.e. which produce a safe, comfortable, and dynamically feasible trajectory from the vehicle's current configuration to the goal configuration.

From 83] it emerges how, a common trait of the automatic steering control solutions for automated driving, is the interaction of preview control, feedback components, and heuristic methods. This is especially evident in [84], where the controller acquires sample values of preview path errors, lateral position error and attitude error, and then generates a steering wheel angle control. In this work the optimal control design is applied to a linear discrete time preview control, although it is still able to deal with 
nonlinear effects induced by high speed manoeuvres.

Moreover, on the related topic of collision avoidance manoeuvres, we find an excellent review of path tracking (and also some path tracking strategies) in 85. In there numerous strategies are evaluated, although no direct conclusion seems possible. In [86] sensor information available about the surrounding environment is used to identify corridors of travel through which the vehicle can safely navigate. Further elaboration on lane change manoeuvres is done in 87$]$. Finally, in 88 the authors presented comprehensive description of constraints imposed for autonomous driving (i.e. traffic lights, velocity limits, forbidden lane-change, lane merging etc.) and demonstrated the first optimal multi-lane driving in urban environment with traffic lights.

Besides the control method, the most important aspect, which can be used to classify the different proposed works, is the model used for the description of the vehicle trajectory. According to [89], there are three main categories in which path tracking algorithms can be classified:

(1) Geometric path tracking methods;

(2) Kinematic model based path tracking methods;

(3) Dynamic model based path tracking methods (not discussed in this paper).

In the following, some interesting approaches are introduced, with special focus on the ones suitable for vehicle path tracking control.

\subsubsection{Geometric path tracking methods}

Geometric path tracking algorithms owe their name to the fact that they exploit geometrical relationships between the desired path to be followed and the model of the vehicle.

The first presented method is the so-called Pure Pursuit algorithm. It is a popular method, widely used in the robotic field over the past few years. In [90], it is used to compute the arc (i.e. the steering angle) necessary to a robot, equipped with an on-board black and white camera, in order to correct its position with respect to the desired one. The obtained results can be extended also to control a four-wheeled vehicle.

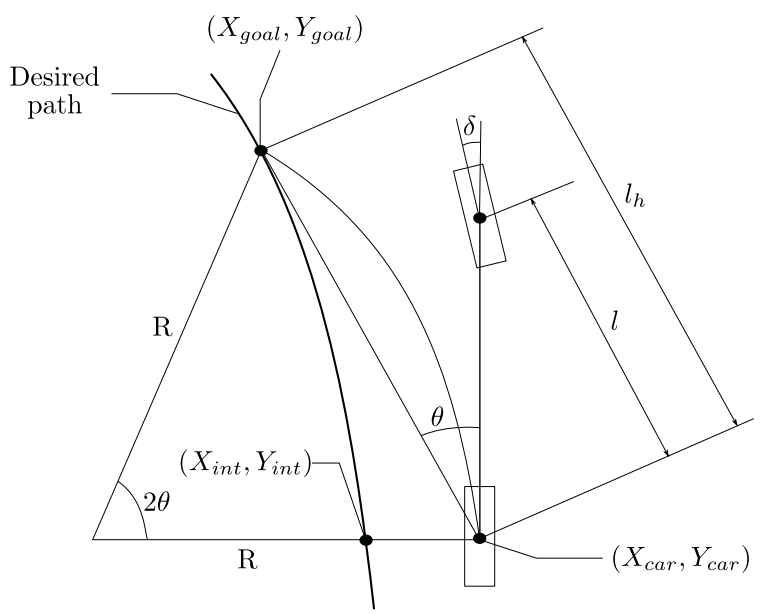

Figure 13.: Pure pursuit model

The Pure Pursuit algorithm exploits a simplified model of the vehicle and its re- 
lationship with the geometrical representation of the desired path. In particular, as described in Fig. 13, a bicycle model of the vehicle is directly used. It is assumed that the vehicle can only move on a 2-D surface and that only the front wheel can steer. Then, having defined the wheelbase of the vehicle $l$, it is straightforward to geometrically derive the equation that links the steering angle $\delta$ and the curvature radius $R$ of the trajectory, followed by the rear wheel, as follows:

$$
\tan \delta=\frac{l}{R}
$$

As described in [91], in its basic implementation, the Pure Pursuit algorithm relies on the following procedure.

Given a constant look-ahead distance $l_{h}$, it is possible to derive the Pure Pursuit control law as follows:

$$
\delta(t)=\tan ^{-1}\left(\frac{2 l \sin (\theta(t))}{l_{h}(t)}\right)
$$

Equation (3) describes the steering angle that must be applied in order to meet the goal point and follow the desired trajectory. In actual implementation, it is a common practice not to use a fixed value of the look-head distance, but a varying one. Then, $l_{h}(t)=k v_{x}(t)$ depends on a tuning parameter $k$ and on the longitudinal velocity of the vehicle, obtaining

$$
\delta(t)=\tan ^{-1}\left(\frac{2 l \sin (\theta)}{k_{P P} v_{x}(t)}\right)
$$

In this manner, a too aggressive control action is avoided in case of high value of $v_{x}$ or small value of the curvature radius as is shown in, for instance, [92]. Then, the control parameter $k_{P P}$ tuning is the key step in order to obtain an effective control action.

In [89], multiple values of $k_{P P}$ are tested for a controller applied to a vehicle that performs common tests, such as a single lane change and the eight course, i.e. an eight-shaped circuit.

By analyzing the test results, it can be stated that:

- for "small" values of $k_{P P}$ (i.e. for a short look-ahead distance) the path tracking is accurate, but the vehicle shows an oscillatory behavior and system instability can arise;

- for "large" values of $k_{P P}$ the system works in stable condition and there are not relevant oscillatory phenomena. However, despite smooth path tracking being guaranteed, it can be inaccurate.

Such results show that a fine tuning of the parameter $k_{P P}$ is needed in order to achieve a proper trade off between tracking performance and stability property.

Since pure-pursuit has been one of the most common path tracking strategy, many studies have been done aimed to improve its performance in actual implementation, for both indoor and outdoor applications. In particular, in [93] the main properties and issues of the pure-pursuit algorithm are studied. On the other hand, in [94, much effort has been spent in analyzing the effect of time delays due to the computational time needed by the hardware components, such as cameras, sensors, etc. 


\subsubsection{Kinematic model based methods}

By employing a kinematic model, all phenomena that occur between the wheels and the road, which give rise to the so-called slipping conditions, are neglected.

This model approximation is used in several works, e.g. in [95], where a survey of these path tracking methods can be found, applied to a rear-wheel driving vehicle.

The interesting control strategy, firstly presented in [96] and further developed in [95], is the so-called Smooth Time-Varying Feedback Control is based on the exploitation of the properties of the so-called kinematic model in path coordinates, used to describe the vehicle motion with respect to the desired path.

This control strategy allows to derive a path tracking time-varying feedback control law. Then, the derived controller must be tuned in order to guarantee the stability of the system and ensure good tracking performance. In 95 also a simple design rule is presented, which guarantees stability while at the same time reducing the number of calibratable parameters.

In [89], several lane change tests have been performed at different velocities, for value of lateral acceleration ranging from 1 to $6 \mathrm{~m} / \mathrm{s}^{2}$. The results of these experiments show that, at low velocities, by increasing lateral acceleration, the path tracking accuracy increase. But at higher velocities (e.g. $20 \mathrm{~m} / \mathrm{s}$ ), the controller performances and robustness drastically decrease. This is due to the fact that this control approach does not take into account some vehicle dynamics that can not be neglected at high velocities.

Kinematic model methods are the most commonly used path tracking approaches adopted in the automotive community. In particular, the trend is to use a cascade structure in which the fist stage consist in the computation of the desired steering angle $\delta$, and the second stage employs an algorithm able to overtake the limits of the kinematic model, which does not take into account the vehicle dynamics. This structure can be found in [97, where two MPC controllers are used for this purpose, or in 98, where a neural-network based algorithm is implemented.

\subsubsection{Considerations for long combination vehicles}

Heavy vehicles, and in particular long combination vehicles, present additional challenges (compared to passenger cars) when designing motion control and path following approaches. Their high centre of mass results in a roll over threshold which that must be considered when calculating the maximum lateral acceleration capability of the vehicle. Typical steady-state roll-over thresholds for a tractor semitrailer vehicle can be as low as $3.5 \mathrm{~m} / \mathrm{s}^{2}$ and vary significantly depending on the payload that is being carried by the vehicle.

The multiple articulation points which are present in a long combination vehicle can also introduce a phenomenon known as rearward amplification; where, depending on the frequency of a steering input on the first vehicle unit, a larger lateral acceleration may be experienced in subsequent vehicle units. A rearward amplification ratio of between 1.1 and 1.3 is typical for a conventional tractor-semitrailer combination 99 , 100. For longer multi-trailer vehicle combinations rearward amplification ratios $>2$ can be exhibited [99]. The maximum rearward amplification ratio for articulated heavy vehicles is typically experienced at frequencies around $0.5 \mathrm{~Hz}$ - such frequencies should therefore be avoided, where possible, when designing path following controllers for articulated heavy vehicles.

Long combination vehicles require significantly more road space to manoeuvre compared to passenger cars; for example, a standard tractor-semitrailer combination can exhibit a swept path width of $>7 \mathrm{~m}$ when navigating a $12.5 \mathrm{~m}$ radius roundabout [99]. 
The swept path of the whole vehicle combination must be taken into account when planning and tracking a path. It should also be considered when selecting a suitable vehicle combination for a given ODD.

The introduction of actively steered axles on trailer and converter-dolly units has been shown to reduce the effects of rearward amplification as well as the overall swept path of long combination vehicles, e.g. in [101] and [102].

The multiple articulation points of long combination vehicles make the task of automated reversing somewhat difficult. Path followers suitable for this task have, however, recently been designed and successfully implemented on multi-trailer combinations, e.g. in 103.

\subsection{Vehicle motion state estimation (VMSE)}

As can be seen from Fig. 12, state-estimation of feedback variables are important for VMC and a comprehensive review of state estimation can be found in [9]. Tire/road friction estimation has attracted particular attention and an extensive review on this model based friction and estimation without tire model is available in [104. One particular challenge in vehicle state and friction estimation is given by small friction utilization: this topic is extensively discussed in 105, 106 for side-slip angle and forces estimation, and in 107 for the road friction. In addition to this, the estimation and compensation of the brake linings coefficient of friction in modern brake-by-wire systems represent another challenging task owing to the stochastic characteristic of the frictional contact dynamics 108. Recent publications on real-time robust identification of tire and brake linings friction can be found in 109, 110] and [111, respectively.

The task for the Vehicle Motion State Estimator (VMSE) is to estimate:

- motion variables of vehicle body, e.g. vehicle speed over ground

- motion variables of axles and wheels, e.g. tire forces

- vehicle parameters, e.g. vehicle mass

- vehicle-environment variables, e.g. road friction

The VMSE fuses vehicle dynamic sensors entities to estimated entities to a desired attribute. Quite often the sensors themselves do not have high enough ASIL and lack of accuracy and precision. The VMSE also gives the confidence of an estimate along with the estimate itself. One important estimate from VMSE is the tire/road friction. What is unique with road friction is that it is crucial for determining the trade-off between risk of accident and speed for the automated vehicle. At the same time, it is also difficult to estimate [112, 113]. In [114 the confusion matrix is presented as a way to understand why road friction is important to know. As seen in 14 if the estimated friction is lower than true one, then we could expect false warnings. These will for a human driven vehicle risk to be experienced as an unwanted disturbance. The selfdriving vehicle will most likely take action and lower the speed to an unacceptable low speed which make the transport task unnecessarily time consuming. If the estimated friction is higher than true one, the self-driving vehicle risks to adapt it's driving style to high road friction, which means that vehicle speed risks to be too high during cornering and time gap to surrounding traffic too small. 


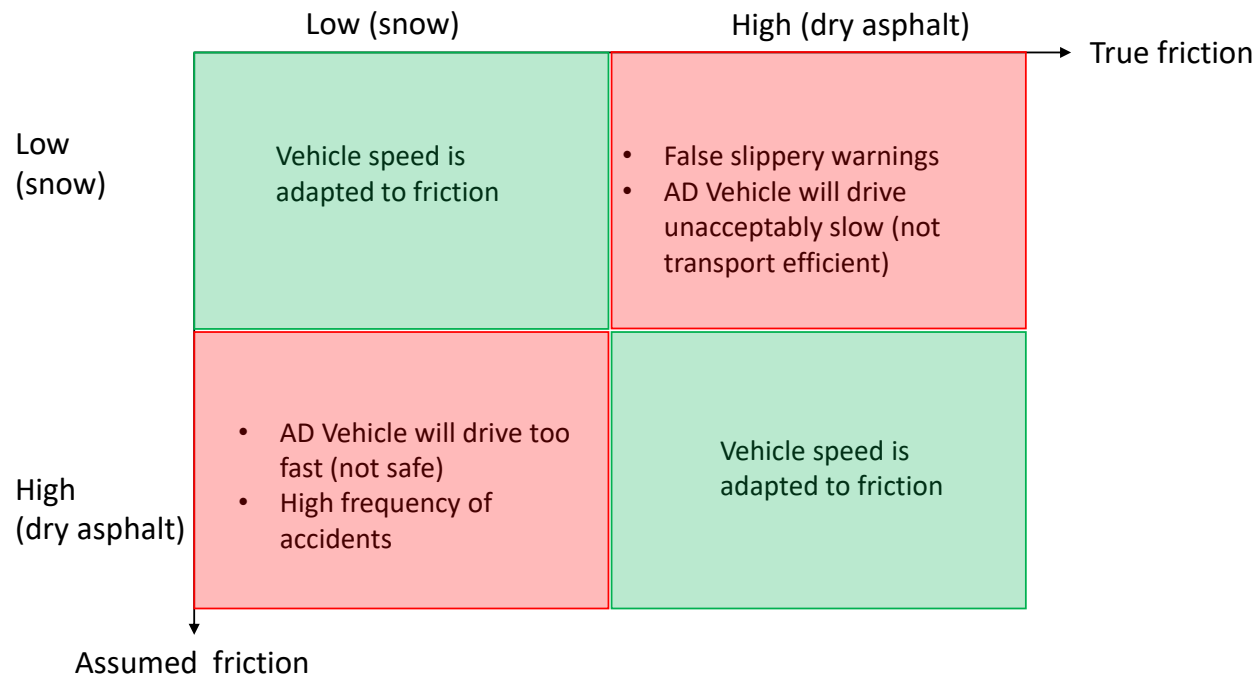

Figure 14.: Confusion matrix of road friction

\subsection{Minimum risk maneuver example}

In case of loss of reference motion, the vehicle may be required to execute a minimum risk manoeuvre and track a minimum risk path. Losses of reference motion can appear as a result of, for example, power blackout, electromagnetic disturbances or communication failures. When such events happen, the vehicle may not be able to operate normally and a minimum risk maneuver could be initiated. Figure 15 shows the use case of a minimum risk maneuver where a safe stop is initiated and controlled. The goal here is to perform a safe stop until standing still, by decelerating and following the last known buffered reference path. One special case of a safe stop is the blind safe stop, where environment sensors are unavailable and the position needs to be controlled blindly by dead-reckoning by for example inertial navigation. Since deadreckoning results in integration drift, there is over time an increasing uncertainty in the position during the control (marked with yellow color in Fig. 15). Since high speed driving results in longer duration of the safe stop, a larger position error is expected at high speeds. Inertial navigation has been applied in many fields. For example for positioning of humans inside buildings [115, 116], mobile telephones [117], under water vehicles [118], aircraft [119], [120] and land-based vehicles [121 126]. Dead reckoning is often used at sea or by aircraft where the Global Navigation Satellite System (GNSS) signal reception is limited. Fig. 16. a shows the filtering results from two different Kalman filter concepts ( $\mathrm{C} 1$ and $\mathrm{C} 2$ ) from [127] using pure dead-reckoning with data from an inertial navigation unit (IMU), wheel speed sensors and steering angle. In Fig. 16.b the position uncertainty is shown. The 2- $\sigma$ ellipses should be geometrically interpreted as the $95 \%$ likelihood of being inside the border. Position from the reference instrumentation (Oxford Technical Solutions RT3000) is also shown together 


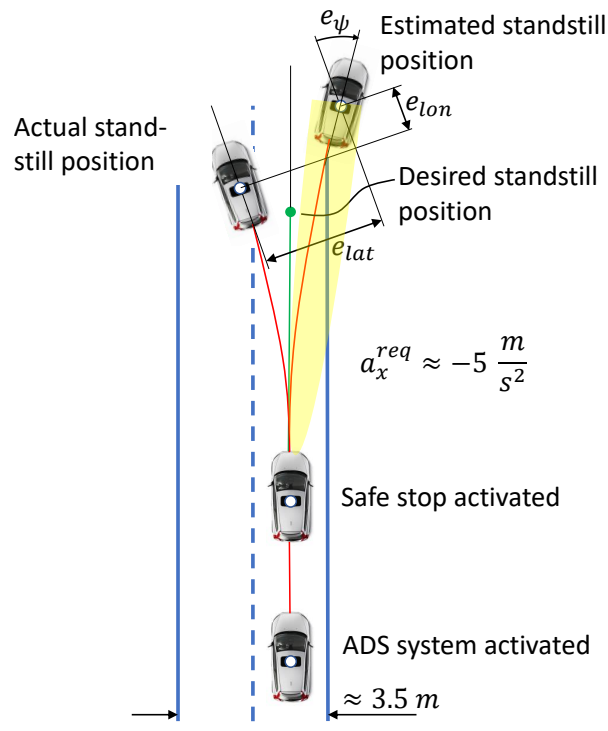

Figure 15.: Example of a minimum risk manoeuvre use case.

with a standard vehicle inbuilt GNSS system. Interestingly, the standard GNSS gives large position errors indicating that a GNSS receiver alone would not solve the problem. The blind safes stop is a particular class of dead-reckoning, where the duration is short (approx. $10 \mathrm{~s}$ ) and the requirement of position precision is high (decimeter laterally). The large uncertainty is a result of a demanding cornering manoeuvre with an initial speed of $120 \mathrm{~km} / \mathrm{h}$. If the position uncertainty from the filtering process can be calculated on-board, the vehicle can adapt it's speed during normal driving to always be able to perform a safe stop within required position constraints. To keep dead reckoning time as short as possible, it would be natural to also decelerate as much as feasible. The risk of rear end collision must however be evaluated which in turn limits too hard deceleration.

In case that also the safe stop algorithm would fail, then the final step is an actuator safe state. This could be executed by the actuators themselves, e.g. locking steering angle and engaging the brakes with a constant brake pressure.

It should also be mentioned that a blind safe stop means that no environment information will be updated until the full stop is reached. Even if the environment information was appropriate just before entering the safe-stop sequence, it is difficult to predict, for instance, all possible variations of motion of the surrounding traffic. The risk for collision is hence increased. The authors believe that protections such as post impact control will therefore be important. After a conceivable first impact, the vehicle occupants are in general more unprotected to a second impact e.g. by already deployed airbags, an unfavorable sitting position which would increase the risk of serious injuries. In case that a first impact is detected, then the vehicle could be controlled to minimize the risk of a secondary impact event, see 128, 129,

\subsection{Redundant steering by differential braking}

Even if a redundant steering actuator is present, as discussed in the previous section, it may be in a degraded state with a reduced capacity. One severe use case is curve driving and loss of steering torque from the steering actuator. Due to the caster trail in 
(a)

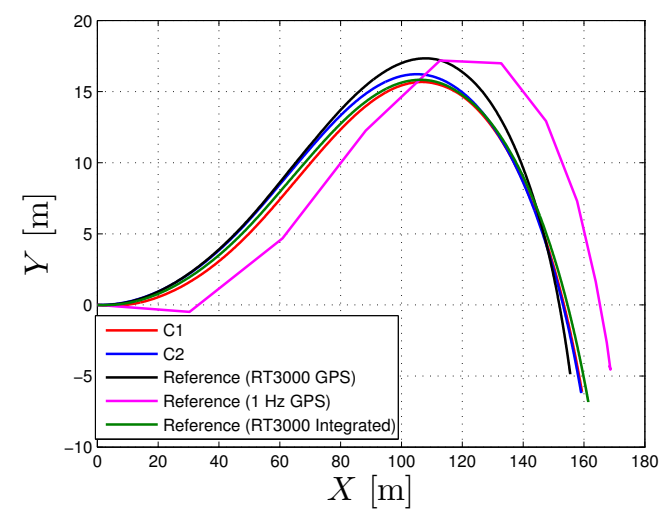

(b)

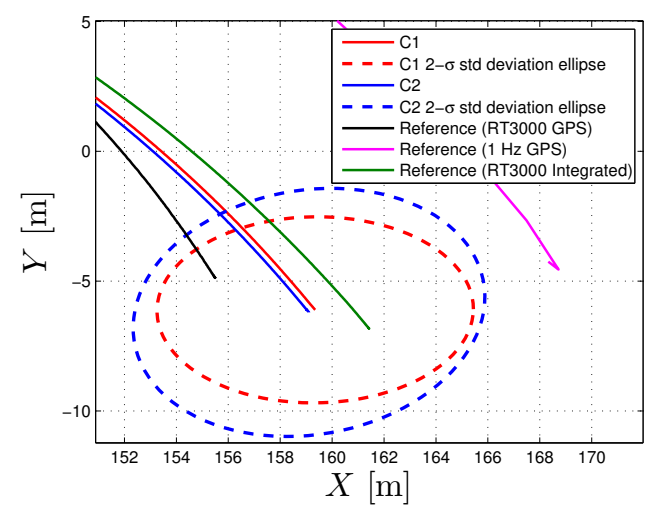

Figure 16.: (a) The estimated X- and Y-positions at $120 \mathrm{~km} / \mathrm{h}$ using dead-reckoning for a blind safe stop. (b) $2-\sigma$ standard deviation ellipses of position

the wheel suspension, the vehicle will quickly leave the lane when the steering torque vanishes. An experiment of loss of steering torque from [13] is seen in Fig. 17. When
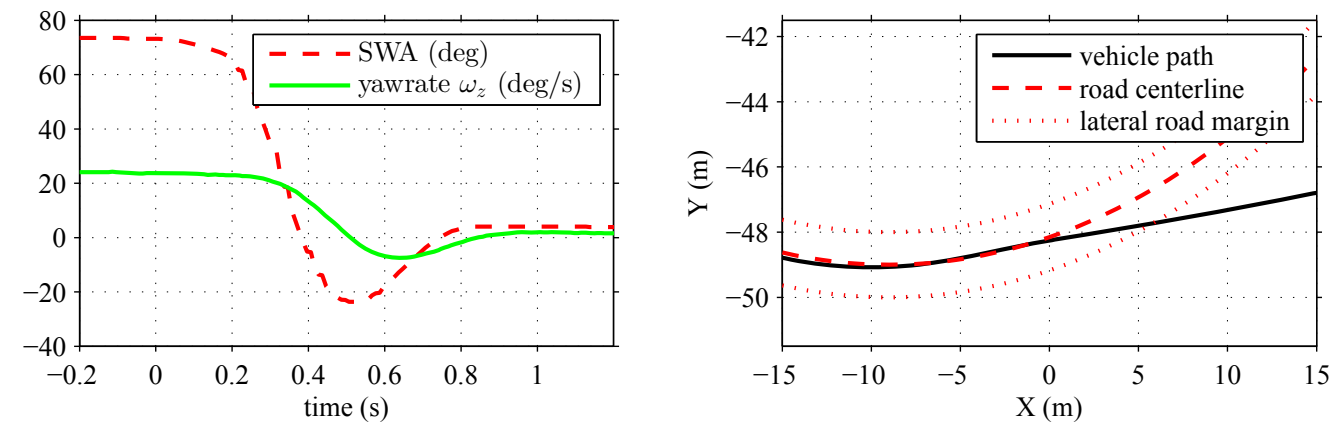

Figure 17.: Effect of vanishing steering torque in a curve with $50 \mathrm{~m}$ radius and 70 $\mathrm{km} / \mathrm{h}$ (from vehicle measurements).

the steering torque from steering actuator disappears, the alignment torque generated at the front axle will induce a change of the steering- wheel angle (SWA) towards zero within approximately $0.8 \mathrm{~s}$. This special case is in the context of self-driving vehicles most likely an unusual case due to the initial high lateral acceleration (approximately $7 \mathrm{~m} / \mathrm{s}^{2}$ ) which is outside any conceivable comfort zone. The case is however selected to demonstrate how quickly the vehicle leaves the road if no control is performed to reduce the effect of the failure. One alternative to overcome the problem with steering actuator degradation is to use differential braking [13] or torque vectoring [130], i.e. applying different wheel torques at left and right side of the vehicle. The method is limited since the vehicle cannot achieve same performance in e.g. lateral acceleration and the solution is not robust 13. For example road friction and wheel suspension parameters such as scrub radius influence the maximum feasible vehicle curvature. Figure 18 shows the results from hard differential braking from at one side of a passenger vehicle. The brake force at left side of the vehicle, $F_{b}^{\text {req }}$, is applied while driving straight ahead. During the brake tests, it was observed that different curvatures, $\rho$, 

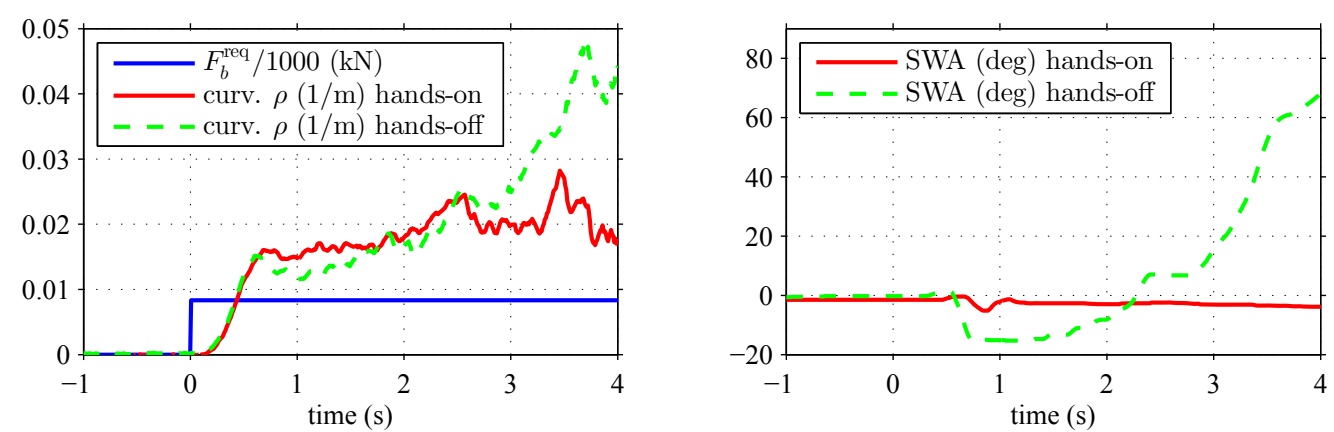

Figure 18.: Experimental response test of hard differential braking curvature (left) and steering-wheel angle (right) for zero steering-wheel angle (hands-on) and zero steering-wheel torque (hands-off) from [13].

(a)

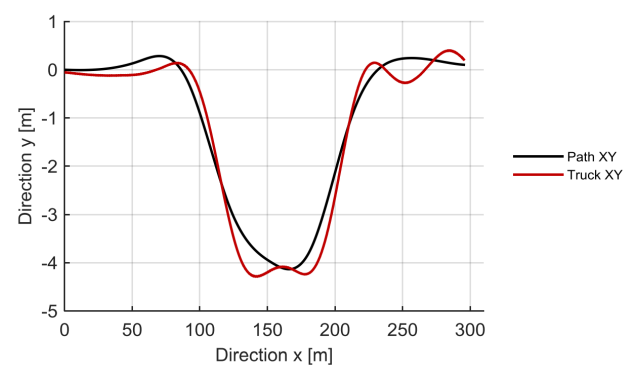

(b)

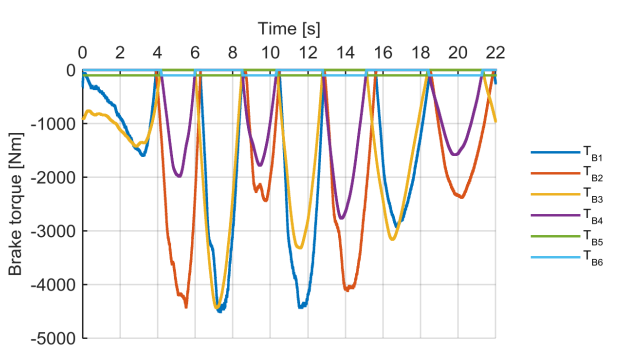

Figure 19.: Lateral control of 3 axle heavy vehicle using steer-by-braking. Double lane change at $60 \mathrm{~km} / \mathrm{h}$, from [131] (a) Path tracking, in $\mathrm{x}$ and y co-ordinates (b) Requested brake torque for each of 6 wheels

were achieved depending on holding hands or not on the steering wheel. When hands were off the steering wheel, the longitudinal tire force on the front left wheel together with the scrub radius generates an alignment torque inducing the steering wheel to, after approximately $1 \mathrm{~s}$, turn anti-clockwise. The induced steering wheel angle, in turn, results in an increased curve radius compared to hold the steering wheel and with a fixed zero angle. In 13 is was found that the suspension design was crucial for the possibility of reach high curvatures. In order to provide a large curvature, the caster angle should be small and the scrub radius large and positive. In addition to the suspension design, it is beneficial to have the center of gravity close the rear axle and to have a large track width.

Steer-by-braking has been investigated as a back-up steering system for automated heavy vehicles, e.g. in [131]. Fig. 19 shows vehicle test results for a 3 axle heavy vehicle, performing an autonomous double-lane change manoeuvre using a steer-bybraking approach. As can be seen in the figure, reasonable path tracking performance can be achieved using differential braking alone on such a vehicle. Such an approach can be used to steer the vehicle for a short period of time, however, for friction braked vehicles, prolonged use of steer-by-braking can result in overheated brake components. It should also be noted that any conventional active steering system(s) fitted to the vehicle must be designed to fail silent for a steer-by-braking controller to be effective. 


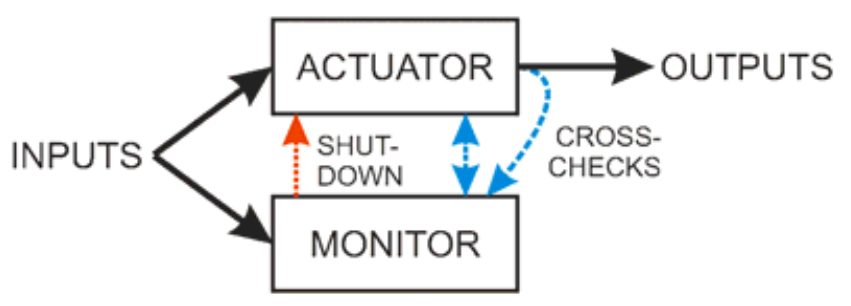

Figure 20.: Monitor/actuator pair conceptual diagram from 134

\section{Verification of Automated Vehicles}

Testing and verification of vehicle systems is always a major part of the development and driving automation systems are no exception. In fact verification might prove one of the most challenging aspects of the development of these systems [132]. In [133 it is shown that automated vehicles would have to be driven hundreds of millions of miles and sometimes hundreds of billions of miles to demonstrate their reliability in terms of fatalities and injuries. This is also noted in [134] where a goal of one fault per $10^{9}$ hours resulting in a violation of a safety goal is not feasible to test the multiple times that time required achieve statistically significant quantification of actual failure rate of the system. Combined this means that significant advances in the research and development of testing and verification methods are required to meet these challenges.

One approach advocated in [134] to mitigate many of the challenges of automated vehicle safety is the use of the monitor/actuator architecture shown in Fig. 20. In such an architecture, functional requirements are allocated separately from system safety requirements. These different sets of requirements are allocated to a actuator and monitor, respectively. The task of the monitor is to override the outputs of the actuator in case of a potential violation of a safety goal. This separation means that, for one, that the two can be developed separately and independently. Also the actuator can be developed with lower integrity than the monitor. Furthermore testing is simplified since system safety testing can be focused on verifying the behavior of the monitor, with less regard to the actuator.

In [134, the method of fault injection is proposed as an important element in the test strategy. This means inserting exceptional conditions and inputs at multiple levels of abstraction to test the robustness of the system and attempt to falsify claims of safety goal fulfillment.

To verify vehicle dynamics control algorithms, [135] proposes (1) the evaluation of the static properties by constrained bifurcation and continuum analysis and (2) the evaluation of the dynamic properties by the optimization-based worst-case seeking. In the first method, the stability of equilibrium points for varying parameters are analyzed, thus creating limits of operation for the analyzed system. In the second approach the optimal control theory is applied to maximize deviations from desired response outputs by seeking the worst possible combination of inputs. These both methods rely on the same underlying system description and are useful to understand the boundaries of the controlled vehicle subsystem from inputs to the VMC and actuator layers.

An approach for the safety verification presented in [136] and [137] is to compute the so-called reachable set of the vehicle. This set then guarantees safety if not intersected by the set of space occupied by other road users. With their approach it is also possible to online compute feasible trajectories for evasive maneuvers.

In [138 a new testing framework is presented that combines the approaches of 
scenario-based testing and functionality-based testing. Based on the so-called semantic diagram which defines the relationship between testing scenarios, tasks, and vehicle functions to measure the sensing/recognition, decision and action behaviors inside a vehicle. This new testing framework is said to have guided parts of the "Intelligent Vehicle Future Challenge" held annually in China.

In [139 machine learning techniques are applied to testing of automated driving as an effective alternative to manual testing of automated driving control. Their approach involves subjecting a controller to an adaptively chosen set of fault scenarios within a simulation environment, and using a genetic algorithm to search for combinations of faults.

All modern testing involves a mix of simulation and physical testing [140]. The benefits of simulation include repeatability, automation and measureability. Furthermore, parameters of simulation models and scenarios can be varied to achieve test coverage that would simply be impossible in physical testing. With validated simulation models [141] even release testing of vehicle variants can be performed [140, 142]. According to a Google report 143 simulation is a vital part of their development of automated vehicles. On the use of simulation they state that:

One benefit of teaching a computer to drive is that it has great memory and recall. With our simulator, we're able to call upon the millions of miles we've already driven and drive those miles again with the updated software. For example, to make left turns at an intersection more comfortable for our passengers, we modified our software to adjust the angle at which our cars would travel. To test this change, we then rerun our entire driving history of $2+$ million miles with the new turning pattern to ensure that it doesn't just make our car better at left turns, but that the change creates a better driving experience overall.

\section{Summary of challenges for automated driving and the connection to the research community}

Figure 21 outlines several challenges that these authors believes need particular attention for autonomous vehicles. The challenges are divided into Vehicle Longitudinal and Lateral Control; Vehicle Motion State Estimation; Robust, Independent and Fault Tolerant Vehicle Systems and finally updated Development Processes.

Many of these aspects are covered in this paper but more research is recommended to be dedicated to study how to obtain a robust and fault-tolerant system and how to reach independence (redundancy) between systems. Some autonomous vehicles, such as robot taxis, are assumed to transport occupants not involved in the driving task meaning that control with high trust and low jerk will be needed. To meet that, novel integrated control that doesn't disturb the driver is likely essential. In addition, motion sickness needs to be considered for planning of route as well as for the longitudinal and lateral control. Here new research is needed to find new predictive control concepts. Moreover, there is need for research in detecting miss-use and wear of the vehicle by means of condition monitoring based on vehicle dynamics knowledge. As an example, a skilled human driver can observe faults in the vehicle's mechanical systems that need actions such as maintenance. The sense for such abilities would be beneficial to automate to lower the cost for faults and increase the life length. Another area of research interest is how to get a logical and smooth transition between the human driver and the autonomous function.

To ensure safety and to not unnecessarily limit the speed of autonomous vehicles, 


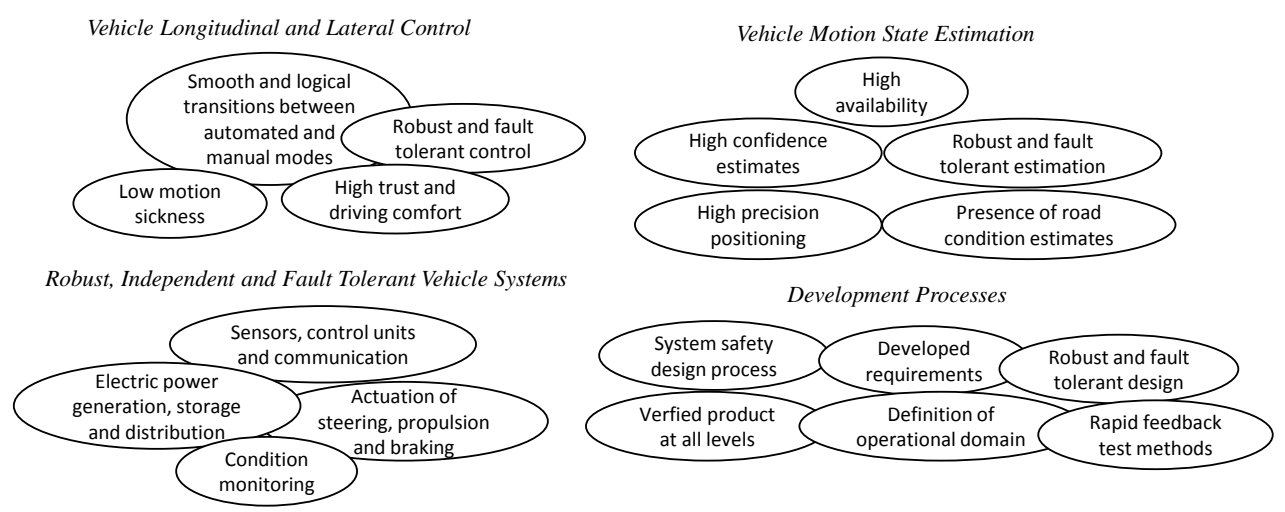

Figure 21.: Challenges in vehicle motion control for automated vehicles.

there is an increasing need for knowing the road condition such as road friction. Since the availability of confident road condition estimates is low, there is likely a need for active excitation of the tires. The question how active tire excitation should be controlled to minimize the influence of comfort has not get much focus in the literature. Also, research on how to use novel sensors, such as microphones, LIDAR, camera etc., for road friction estimation will become essential and how to adapt sensor fusion technologies by using Extended Kalman Filter, Unscented Kalman filter and particle filters. Another area of research interest is high precision positioning and focus on how to estimate the position of the vehicle.

For system safety, there a need for research on how to apply an interpret safety requirements. Safety requirements are typically defined at a customer function level, but attention should be paid on how to brake down requirements across the vehicle motion control architecture. Further on, little research has been dedicated to the question how to verify requirements allocated to the architecture and how to limit the operational domain to fulfill them.

\section{Conclusions}

In this paper we discussed how the vehicle control is affected by automated driving on public roads. In addition we also described the redundancy needed for fault tolerant operation, for a road vehicle meet certain safety goals. Firstly general considerations for automated driving on public roads were discussed. Here we illustrated the importance of the relaying of status and capability of each part of the driving automation system. Secondly the concept of system safety as well as system solutions to fault tolerant actuation of steering and braking and the associated fault tolerant power supply was described. Notably the question of restricting the operational domain of the vehicle in order to be able to proceed with reduced safety integrity in case of reduced capability on any part of the driving automation system was discussed. In this area, the authors also call for more research on applications of this principle. Thirdly we expanded on the aspects of path tracking, state estimation of vehicle motion control required for automated driving as well as an example of a minimum risk maneuver and redundant steering by means of differential braking. The steering by differential braking could offer heterogeneous or dissimilar redundancy that complements the redundancy of 
described fault tolerant steering systems for driving automation equipped vehicles, but more studies in this area is also warranted. Finally the important topic of verification of driving automation systems was addressed. Verification of driving automation systems is a critical topic that requires more research and development in order to realize the promises of driving automation to its users and society as a whole through improved traffic safety, fuel efficiency, traffic flow and more.

\section{Acknowledgements}

The research activities are performed as a part of the Interdisciplinary Training Network in Multi-Actuated Ground Vehicles (ITEAM) project in the European Union's Horizon 2020 research and innovation program under Marie Skłodowska-Curie Grant Agreement No. 675999.

\section{Nomenclature}

AD Automated driving

ADAS Advanced driving assistance systems

ADS Automated driving system

ASIL Automotive safety integrity level

BEV Battery electric vehicle

CAN Controller area network

DDT Dynamic driving task

EPAS Electric power assisted steering

EPS Electric power steering

FHSS Frequency-hopping spread spectrum

FIT Failure in time

GNSS Global navigation satellite system

HEV Hybrid electric vehicle

HV High voltage

ICE Internal combustion engine

IMU Inertial measurement unit

LIDAR Light detection and ranging

LKA Lane keeping assist

MPC Model predictive control

ODD Operational driving domain

OEDR Object and event detection and response

PDC Power distribution center

PHEV Plugin hybrid electric vehicle

RTI Request to intervene

SOTIF Safety of the intended function

SWA Steering wheel angle

VMC Vehicle motion control

VMSE Vehicle motion state estimation 


\section{References}

[1] Montanaro U, Dixit S, Fallah S, et al. Towards connected autonomous driving: review of use-cases. Vehicle System Dynamics. 2018;

[2] Fagnant DJ, Kockelman K. Preparing a nation for autonomous vehicles: opportunities, barriers and policy recommendations. Transportation Research Part A: Policy and Practice. 2015 7;77:167-181. Available from: https://www . sciencedirect.com/science/article/pii/S0965856415000804.

[3] SAE J3016. Taxonomy and Definitions for Terms Related to Driving Automation Systems for On-Road Motor Vehicles. SAE International; 2018.

[4] Zhankaziev S. Current Trends of Road-traffic Infrastructure Development. Transportation Research Procedia. 2017 1;20:731-739. Available from: https: //www.sciencedirect.com/science/article/pii/S2352146517301187.

[5] Tsugawa S, Jeschke S, Shladover SE. A Review of Truck Platooning Projects for Energy Savings. IEEE Transactions on Intelligent Vehicles. 2016;1(1):68-77.

[6] Dey KC, Yan L, Wang X, et al. A Review of Communication, Driver Characteristics, and Controls Aspects of Cooperative Adaptive Cruise Control (CACC). IEEE Transactions on Intelligent Transportation Systems. 2016;17(2):491-509.

[7] Bergenhem C, Pettersson H, Coelingh E, et al. Overview of Platooning Systems. In: Proceedings of the 19th ITS World Congress, Oct 22-26, Vienna, Austria; 2012 .

[8] Karlsson K, Carlsson J, Larsson M, et al. Evaluation of the V2V channel and diversity potential for platooning trucks. In: 2016 10th European Conference on Antennas and Propagation (EuCAP); 2016. p. 1-5.

[9] Guo H, Cao D, Chen H, et al. Vehicle dynamic state estimation: State of the art schemes and perspectives. IEEE/CAA Journal of Automatica Sinica. 2018; $5(2): 418-431$.

[10] Sklaroff JR. Redundancy Management Technique for Space Shuttle Computers. IBM Journal of Research and Development. 1976;20(1):20-28.

[11] Tomayko JE. Achieving Reliability: The Evolution of Redundancy in American Manned Spacecraft. Journal of the British Interplanetary Society. 1985;38:545553.

[12] Stanton NA, Marsden P. From fly-by-wire to drive-by-wire: Safety implications of automation in vehicles. Safety Science. 1996;

[13] Jonasson M, Thor M. Steering redundancy for self-driving vehicles using differential braking. Vehicle System Dynamics. 2018 5;56(5):791-809. Available from: https://doi.org/10.1080/00423114.2017.1356929.

[14] Bengler K, Dietmayer K, Farber B, et al. Three decades of driver assistance systems: Review and future perspectives. IEEE Intelligent Transportation Systems Magazine. 2014;6(4):6 - 22.

[15] Gordon TJ, Lidberg M. Automated driving and autonomous functions on road vehicles. Vehicle System Dynamics. 2015;53(7: State of the Art papers of the 24th IAVSD Symposium):958-994.

[16] Katrakazas C, Quddus M, Chen WH, et al. Real-time motion planning methods for autonomous on-road driving: State-of-the-art and future research directions. Transportation Research Part C: Emerging Technologies. 2015;

[17] Brännstrom M, Sandblom F, Hammarstrand L. A probabilistic framework for decision-making in collision avoidance systems. IEEE Transactions on Intelligent Transportation Systems. 2013;.

[18] Brännström M, Coelingh E, Sjöberg J. Model-based threat assessment for avoid- 
ing arbitrary vehicle collisions. IEEE Transactions on Intelligent Transportation Systems. 2010;

[19] Campbell S, O'Mahony N, Krpalcova L, et al. Sensor Technology in Autonomous Vehicles : A review. In: 2018 29th Irish Signals and Systems Conference (ISSC); 2018. p. 1-4.

[20] Yang X, Liu L, Vaidya NH, et al. A vehicle-to-vehicle communication protocol for cooperative collision warning. In: The First Annual International Conference on Mobile and Ubiquitous Systems: Networking and Services, 2004. MOBIQUITOUS 2004.; 2004. p. 114-123.

[21] Leblanc H, Horst F. Communication architecture for a locomotive remote control system ; 2004.

[22] Construction Week. Volvo's real-life remote-controlled construction truck ; 2018. Available from: https://youtu.be/2GEYSfi5gWQ

[23] Isermann R, Schwarz R, Stolzl S. Fault-tolerant drive-by-wire systems. IEEE Control Systems Magazine. 2002;22(5):64-81.

[24] Vivas-López CA, Hernández-Alcantara D, Tudón-Martínez JC, et al. Review on Global Chassis Control. IFAC Proceedings Volumes. 2013;46(2):875 - 880.

[25] Kissai M, Monsuez B, Tapus A. Review of integrated vehicle dynamics control architectures. In: 2017 European Conference on Mobile Robots (ECMR); Sep.; Paris. IEEE; 2017. p. 1-8.

[26] Yu F, Li DF, Crolla DA. Integrated Vehicle Dynamics Control - State-of-the Art Review. In: 2008 IEEE Vehicle Power and Propulsion Conference; 9; Harbin, Hei Longjiang, China. IEEE; 2008. p. 1-6.

[27] Moon S, Cho W, Yi K. Intelligent vehicle safety control strategy in various driving situations. In: Vehicle System Dynamics; 2010.

[28] Taş, Kuhnt F, Zöllner JM, et al. Functional system architectures towards fully automated driving. In: 2016 IEEE Intelligent Vehicles Symposium (IV); 2016. p. 304-309.

[29] Kritayakirana K, Gerdes JC. Autonomous vehicle control at the limits of handling. International Journal of Vehicle Autonomous Systems. 2012;

[30] Boada BL, Boada MJ, Díaz V. Fuzzy-logic applied to yaw moment control for vehicle stability. Vehicle System Dynamics. 2005;

[31] Werling M, Reinisch P, Gröll L. Robust power-slide control for a production vehicle. International Journal of Vehicle Autonomous Systems. 2015;

[32] Acosta M, Kanarachos S, Blundell M. Vehicle Agile Maneuvering: From Rally Drivers to a Finite State Machine Approach. In: 2016 IEEE Symposium Series on Computational Intelligence (SSCI); 12. IEEE; 2016. p. 1-8.

[33] Acosta M, Kanarachos S, Fitzpatrick ME. A Hybrid Hierarchical Rally Driver Model for Autonomous Vehicle Agile Maneuvering on Loose Surfaces. In: 14th International Conference on Informatics in Control, Automation and Robotics; Madrid, Spain; 2017. p. 216-225.

[34] Acosta M, Kanarachos S. Teaching a Vehicle to Autonomously Drift: A DataBased Approach Using Neural Networks. Knowledge-Based Systems. 2018 8; 153:12-28.

[35] Yang X, Chen S. Non-linear dynamics mechanism of road vehicle at losing stability in critical cornering situations. Int J Vehicle Autonomous Systems. 2014; 12(2):116-140.

[36] Bobier-Tiu CG, Beal CE, Kegelman JC, et al. Vehicle control synthesis using phase portraits of planar dynamics. Vehicle System Dynamics. 2018;

[37] Horiuchi S, Okada K, Nohtomi S. Analysis of accelerating and braking sta- 
bility using constrained bifurcation and continuation methods. Vehicle System Dynamics. 2008;46(sup1):585-597. Available from: https://doi.org/10.1080/ 00423110802007779 .

[38] Klomp M, Lidberg M, Gordon T. On optimal recovery from terminal understeer. Proceedings of the Institution of Mechanical Engineers, Part D: Journal of Automobile Engineering. 2014;228(4).

[39] Fors V, Olofsson B, Nielsen L. Formulation and interpretation of optimal braking and steering patterns towards autonomous safety-critical manoeuvres. Vehicle System Dynamics. 2018;

[40] Arikere A, Yang D, Klomp M, et al. Integrated evasive manoeuvre assist for collision mitigation with oncoming vehicles. Vehicle System Dynamics. 2018;

[41] Arikere A, Yang D, Klomp M. Optimal motion control for collision avoidance at Left Turn Across Path/Opposite Direction intersection scenarios using electric propulsion. Vehicle System Dynamics. 2018;

[42] Horiuchi S. Evaluation of chassis control method through optimisation-based controllability region computation. Vehicle System Dynamics. 2012;50(sup1):1931. Available from: https://doi.org/10.1080/00423114.2012.657653.

[43] Klomp M. Longitudinal force distribution using quadratically constrained linear programming. Vehicle System Dynamics. 2011;49(12).

[44] Wanner D, Trigell AS, Drugge L, et al. Survey on fault-tolerant vehicle design. In: 26th Electric Vehicle Symposium, (EVS26); Los Angeles; 2012.

[45] ISO 26262. Road vehicles - Functional safety ; 2018. Available from: https: //www. iso.org/standard/68383.html.

[46] Eckert A, Hartmann BJ, Sevenich M, et al. Emergency Steer \&amp; Brake Assist - a Systematic Approach for System Integration of Two Complementary Driver Assistance Systems; 2011.

[47] Cerone V, Milanese M, Regruto D. Combined Automatic Lane-Keeping and Driver's Steering Through a 2-DOF Control Strategy. IEEE Transactions on Control Systems Technology. 2009;17(1):135-142.

[48] Uhlemann E. Connected-Vehicles Applications Are Emerging. IEEE Vehicular Technology Magazine. 2016;11(1):25-96.

[49] Lee Jw, Lee YH, Litkouhi BB. Driver hands on/off detection during automated lane centering/changing maneuver ; 2009. Available from: https://patents . google.com/patent/US20100228417A1/en.

[50] Vicente F, Huang Z, Xiong X, et al. Driver Gaze Tracking and Eyes off the Road Detection System. IEEE Transactions on Intelligent Transportation Systems. 2015;

[51] Lie A, Tingvall C, Krafft M, et al. The Effectiveness of Electronic Stability Control (ESC) in Reducing Real Life Crashes and Injuries. Traffic Injury Prevention. 2006 3;7(1):38-43. Available from: https://doi.org/10.1080/ 15389580500346838.

[52] ISO 26262-9:2018. Road vehicles - Functional safety - Part 9: Automotive safety integrity level (ASIL)-oriented and safety-oriented analyses. ISO; 2018.

[53] Gebauer C. Fail Operational and ISO 26262, 2nd Edition. In: Functional Safety in Automotive; Stuttgart-Fellbach; 2018.

[54] ISO/PAS 21448:2019. Road vehicles - Safety of the intended functionality. International Organization for Standardization; 2019. Available from: https: //www. iso.org/standard/70939.html.

[55] Altby A, Majdandzic D. Design and implementation of a fault-tolerant drive-by-wire system. Gothenburg: Chalmers University of Technology; 2014. 
Available from: http://publications.lib.chalmers.se/records/fulltext/ 208025/208025.pdf.

[56] Horiuchi S, Okada K, Nohtomi S. Stochastic robustness analysis and synthesis of nonlinear vehicle dynamics controller. Vehicle System Dynamics. 2002;37(sup1):183-196. Available from: https://doi.org/10.1080/00423114. 2002.11666231 .

[57] Nohtomi S, Okada K, Horiuchi S. Application of analytic hierarchy process to stochastic robustness synthesis of integrated vehicle controllers. Vehicle System Dynamics. 2004;42(1-2):3-21. Available from: https://doi.org/10.1080/ 00423110412331291571.

[58] Nohtomi S, Okada K, Urabe H, et al. Simultaneous robust optimization of suspension and active control system of road vehicles for handling improvement. Vehicle System Dynamics. 2006;44(sup1):904-912. Available from: https: //doi.org/10.1080/00423110600907550

[59] Isermann R, Schwarz R, Stoelzl S. Fault-Tolerant Drive-by-Wire Systems. In: "IEEE Control Systems Magazine"; 2002.

[60] Rogova E, Lodewijks G. Methods of reliability assessment of heterogeneous redundant systems. IFAC-PapersOnLine. 2016;49(12):139-144.

[61] Gonzalez-Prieto I, Duran MJ, Barrero FJ. Fault-Tolerant Control of Six-Phase Induction Motor Drives with Variable Current Injection. IEEE Transactions on Power Electronics. 2017;

[62] KUANG X, GUO H, XU J, et al. Research on a six-phase permanent magnet synchronous motor system at dual-redundant and fault tolerant modes in aviation application. Chinese Journal of Aeronautics. 2017 8;30(4):1548 1560. Available from: https://www.sciencedirect.com/science/article/ pii/S1000936117301048.

[63] Welchko BA, Lipo TA, Jahns TM, et al. Fault tolerant three-phase AC motor drive topologies; A comparison of features, cost, and limitations. In: IEMDC 2003 - IEEE International Electric Machines and Drives Conference; 2003.

[64] Nilsson P. Redundant Brake Actuators For Fail Safe Brake System ; 2007.

[65] Jörn Albers and Ingo Koch. Reliability of Lead-Acid Batteries. In: Elektrik/Elektronik in Hybrid- und Elektrofahrzeugen und elektrisches Energiemanagement VII; 2016.

[66] Jörg Kammermann and Igor Bolvashenkov and Stefan Schwimmbeck and HansGeorg Herzog. Reliability of Induction Machines: Statistics, Tendencies, and Perspectives. In: Proceedings of the IEEE 26th International Symposium on Industrial Electronics; 2017.

[67] ISO 6469-3:2018. Electrically propelled road vehicles - Safety specifications Part 3: Electrical safety. ISO; 2018.

[68] Driving into 2025: The Future of Electric Vehicles https://www.jpmorgan . com/global/research/electric-vehicles; 2018.

[69] Graf A, Vogel D, Gantioler J, et al. Intelligente Leistungshalbleiter für zukünftige Kfz-Bordnetze. In: 17. Tagung "Elektronik im Kraftfahrzeug"; 1997.

[70] Reif K, editor. Batterien, Bordnetze und Vernetzung. Vieweg + Teubner Verlag; 2014.

[71] Reif K, editor. Automobilelektronik. Springer Vieweg; 2014.

[72] Short M, Pont MJ. Fault-tolerant time-triggered communication using CAN. IEEE Transactions on Industrial Informatics. 2007;

[73] Davis RI, Burns A, Bril RJ, et al. Controller Area Network (CAN) schedulability analysis: Refuted, revisited and revised. Real-Time Systems. 2007;35(3):239-272. 
[74] Kopetz H, Grunsteidl G. TTP - A time-triggered protocol for fault-tolerant real-time systems. In: FTCS-23 The Twenty-Third International Symposium on Fault-Tolerant Computing; 1993. p. 524-533.

[75] Arora A, Masud Mahmud S. Performance Analysis of Fault Tolerant TTCAN System; 2005.

[76] ISO 26262-5:2018. Road vehicles - Functional Safety - Part 5: Product development at the hardware level. ISO; 2011.

[77] Hommel M. Fault-tolerant electromechanical steer-by-wire steering actuator ; 2001.

[78] Grabs P, Plaschke M, Heuermann M. E/E Architecture Proposals for Automated Driving and their Failure Robustness. In: Proceedings of the Electric and Electronic Systems in Hybrid and Electric Vehicles and Electrical Energy Management; 2015.

[79] Schumi S. Energy and Supply Concepts for Automated Driving. In: "Proceedings of the IEEE Conference on ESARS ITEC"; 2018.

[80] Albus J. A reference model architecture for intelligent unmanned ground vehicles. In: Proceedings of the SPIE 16th Annual International Symposium on Aerospace/Defense Sensing, Simulation and Controls; Citeseer; 2002. p. 303310.

[81] Madhavan R, Messina ER, Albus JS. Intelligent vehicle systems: A 4d/rcs approach. Book Nova Science Publishers Inc.; 2006.

[82] Amer NH, Zamzuri H, Hudha K, et al. Modelling and Control Strategies in Path Tracking Control for Autonomous Ground Vehicles: A Review of State of the Art and Challenges. Journal of Intelligent and Robotic Systems: Theory and Applications. 2017;86(2).

[83] Paden B, Ćáp M, Yong SZ, et al. A survey of motion planning and control techniques for self-driving urban vehicles. IEEE Transactions on intelligent vehicles. 2016;1(1):33-55.

[84] Sharp R, Casanova D, Symonds P. A mathematical model for driver steering control, with design, tuning and performance results. Vehicle system dynamics. 2000;33(5):289-326.

[85] Zakir U, Hamid A, Saito Y, et al. A review on threat assessment, path planning and path tracking strategies for collision avoidance systems of autonomous vehicles. Int J Vehicle Autonomous Systems. 2018;14(2):134-169.

[86] Constantin A, Park J, Iagnemma K. A margin-based approach to threat assessment for autonomous highway navigation. In: IEEE Intelligent Vehicles Symposium, Proceedings; 2014.

[87] Nilsson J, Silvlin J, Brannstrom M, et al. If, When, and How to Perform Lane Change Maneuvers on Highways. IEEE Intelligent Transportation Systems Magazine. 2016;.

[88] Ajanovic Z, Lacevic B, Shyrokau B, et al. Search-based optimal motion planning for automated driving. In: 2018 IEEE/RSJ International Conference on Intelligent Robots and Systems (IROS); IEEE; 2018. p. 4523-4530.

[89] Snider JM, et al. Automatic steering methods for autonomous automobile path tracking. Robotics Institute, Pittsburgh, PA, Tech Rep CMU-RITR-09-08. 2009;

[90] Wallace R, Stentz AT, Thorpe C, et al. First results in robot road-following. In: Proceedings of the International Joint Conference on Artificial Intelligence; January; 1985.

[91] Samuel M, Hussein M, Binti M. A review of some pure-pursuit based path track- 
ing techniques for control of autonomous vehicle. International Journal of Computer Applications. 2016 02;135:35-38.

[92] Klomp M, Olsson K, Sandberg C. Non-linear steering control for limit handling conditions using preview path curvature. International Journal of Vehicle Autonomous Systems. 2014;12(3):266-283.

[93] Coulter RC. Implementation of the pure pursuit path tracking algorithm. Pittsburgh, PA: Robotics Institute , Carnegie Mellon University; 1992. CMU-RI-TR92-01.

[94] Murphy KN. Analysis of robotic vehicle steering and controller delay. In: 5th International Symposium on Robotics and Manufacturing; August; 1994.

[95] De Luca A, Oriolo G, Samson C. Feedback control of a nonholonomic car-like robot. In: Robot motion planning and control. Springer; 1998. p. 171-253.

[96] Samson C. Control of chained systems application to path following and timevarying point-stabilization of mobile robots. IEEE transactions on Automatic Control. 1995;40(1):64-77.

[97] Raffo GV, Gomes GK, Normey-Rico JE, et al. A predictive controller for autonomous vehicle path tracking. IEEE transactions on intelligent transportation systems. 2009;10(1):92-102.

[98] Fierro R, Lewis FL. Control of a nonholonomic mobile robot using neural networks. IEEE transactions on neural networks. 1998;9(4):589-600.

[99] Aurell J, Wadman T. Vehicle combinations based on the modular concept. Nordiska Vägtekniska Förbundet; 2007. ISSN 0347-2485.

[100] Cheng C, Cebon D. Improving roll stability of articulated heavy vehicles using active semi-trailer steering. Vehicle System Dynamics. 2008;46(sup1):373-388. Available from: https://doi.org/10.1080/00423110801958576.

[101] Roebuck R, Odhams A, Tagesson K, et al. Implementation of trailer steering control on a multi-unit vehicle at high speeds. J Dyn Sys, Meas, Control. 2008; $136(2)$.

[102] Islam MM, Laine L, Jacobson B. Improve safety by optimal steering control of a converter dolly using particle swarm optimization for low-speed maneuvers. In: 2015 IEEE 18th International Conference on Intelligent Transportation Systems; Sep.; 2015. p. 2370-2377.

[103] Rimmer AJ, Cebon D. Theory and practice of reversing control on multiplyarticulated vehicles. Proceedings of the Institution of Mechanical Engineers, Part D: Journal of Automobile Engineering. 2016;230(7):899-913. Available from: https://doi.org/10.1177/0954407015596918.

[104] Acosta M, Kanarachos S, Blundell M. Virtual Tyre Force Sensors: An Overview of Tyre Model-Based and Tyre Model-Less State Estimation Techniques. In: Proceedings of the Institution of Mechanical Engineers, Part D: Journal of Automobile Engineering; 10; 2017.

[105] van Aalst S, Naets F, Boulkroune B, et al. An adaptive vehicle sideslip estimator for reliable estimation in low and high excitation driving. IFAC-PapersOnLine. 2018;51(9):243-248.

[106] Regolin E, Alatorre Vazquez AG, Zambelli M, et al. A sliding mode virtual sensor for wheel forces estimation with accuracy enhancement via ekf. IEEE Transactions on Vehicular Technology. 2019;

[107] Acosta M, Kanarachos S, Blundell M. Road Friction Virtual Sensing: A Review of Estimation Techniques with Emphasis on Low Excitation Approaches. Applied Sciences. 2017 11;7(12).

[108] Eriksson M, Bergman F, Jacobson S. On the nature of tribological contact in 
automotive brakes. Wear. 2002;252(1-2):26-36.

[109] Regolin E, Ferrara A. Svm classification and kalman filter based estimation of the tire-road friction curve. IFAC-PapersOnLine. 2017;50(1):3382-3387.

[110] Sharifzadeh M, Senatore A, Farnam A, et al. A real-time approach to robust identification of tyre-road friction characteristics on mixed-mu roads ; 2018.

[111] Ricciardi V, Savitski D, Augsburg K, et al. Estimation of brake friction coefficient for blending function of base braking control. SAE International Journal of Passenger Cars-Mechanical Systems. 2017;10(2017-01-2520):774-785.

[112] Lex C. Estimation of the maximum coefficient of friction between tire and road based on vehicle state measurements [dissertation]; 2015.

[113] Gustafsson F. Slip-based tire-road friction estimation. Automatica. 1997; 33(6):1087-1099.

[114] Jonasson M, Ohlsson N, Chowdhury SR, et al. Automated road friction estimation using car-sensor suite: Machine learning approach [URL: https://www . researchgate.net/publication/328163474_Automated_Road_Friction_ Estimation_using_Car-sensor_Suite_MachineLearning_Approach|; 2018.

[115] Ojeda L, Borenstein J. Personal dead-reckoning system for gps-denied environments. In: 2007 IEEE International Workshop on Safety, Security and Rescue Robotics; Sept; 2007. p. 1-6.

[116] Fang L, Antsaklis PJ, Montestruque LA, et al. Design of a wireless assisted pedestrian dead reckoning system - the navmote experience. IEEE Transactions on Instrumentation and Measurement. 2005 Dec;54(6):2342-2358.

[117] F Evennou FM. Advanced integration of wifi and inertial navigation systems for indoor mobile positioning. Journal on Applied Signal Processing. 2006;:1-11.

[118] Grenon G, An PE, Smith SM, et al. Enhancement of the inertial navigation system for the morpheus autonomous underwater vehicles. IEEE Journal of Oceanic Engineering. 2001 Oct;26(4):548-560.

[119] Berman Z, Powell JD. The role of dead reckoning and inertial sensors in future general aviation navigation. In: IEEE 1998 Position Location and Navigation Symposium (Cat. No.98CH36153); April; 1998. p. 510-517.

[120] Barshan B, Durrant-Whyte HF. Inertial navigation systems for mobile robots. IEEE Transactions on Robotics and Automation. 1995 June;11(3):328-342.

[121] Bevly DM, Parkinson B. Cascaded kalman filters for accurate estimation of multiple biases, dead-reckoning navigation, and full state feedback control of ground vehicles. IEEE Transactions on Control Systems Technology. 2007 March; 15(2):199-208.

[122] Carlson C. Estimation with applications for automobile dead reckoning and control. In: PhD Thesis, Stanford University, US; 2004. p. 1-6.

[123] Martucci F. On-board recursive state estimation for dead-reckoning in an autonomous truck. In: Master Thesis, KTH, Sweden; 2014. p. 1-6.

[124] Lezniak TW, Lewis RA, McMillen RA. A dead reckoning/map correlation system for automatic vehicle tracking. IEEE Transactions on Vehicular Technology. 1977;26:47-60.

[125] Kao WW. Integration of gps and dead-reckoning navigation systems. In: Vehicle Navigation and Information Systems Conference, 1991; Vol. 2; Oct; 1991. p. 635-643.

[126] Dissanayake G, Sukkarieh S, Whyte HD. The aiding of a low-cost strapdown inertial measurement unit using vehicle model constraints for land vehicle applications. IEEE Transactions on Robotics and Automation. 2001;17(5).

[127] Jonasson M, Rogenfelt Lanfelt C, et al. Inertial navigation and position uncer- 
tainty during a blind safe stop of an autonomous vehicle. submitted to IEEE Transactions on Vehicular Technology. 2019;

[128] D Yang BJ T J Gordon, Jonasson M. Quasi-linear optimal path controller applied to post impact vehicle dynamics. IEEE Transaction on Intelligent Transportation System. 2012;13(4).

[129] Yang D, Gordon TJ, Jacobson B, et al. Optimized brake-based control of path lateral deviation for mitigation of secondary collisions. Proceedings of the Institution of Mechanical Engineers, Part D: Journal of Automobile Engineering. 2011;225(12):1587-1604. Available from: https://doi.org/10.1177/ 0954407011412856

[130] Raksincharoensak P, Nagai M, Shino M. Lane keeping control strategy with direct yaw moment control input by considering dynamics of electric vehicle. Vehicle System Dynamics. 2006;

[131] Volz P. Analysis of Potential in Asymmetric Braking for Autonomous Applications ; 2018.

[132] Åsljung D, Nilsson J, Fredriksson J. Validation of collision frequency estimation using extreme value theory. In: IEEE Conference on Intelligent Transportation Systems, Proceedings, ITSC; 2018.

[133] Kalra N, Paddock SM. Driving to safety: How many miles of driving would it take to demonstrate autonomous vehicle reliability? Transportation Research Part A: Policy and Practice. 2016 12;94:182-193. Available from: https://www . sciencedirect.com/science/article/pii/S0965856416302129.

[134] Koopman P, Wagner M. Challenges in Autonomous Vehicle Testing and Validation. SAE International Journal of Transportation Safety. 2016;

[135] Horiuchi S, Okada K, Nohtomi S. Model-based validation procedure for the certification of advanced chassis control systems. Vehicle System Dynamics. 2010;48(sup1):393-409. Available from: https://doi.org/10.1080/ 00423111003777780 .

[136] Althoff M, Althoff D, Wollherr D, et al. Safety verification of autonomous vehicles for coordinated evasive maneuvers. In: 2010 IEEE Intelligent Vehicles Symposium; 2010. p. 1078-1083.

[137] Althoff M, Dolan JM. Online Verification of Automated Road Vehicles Using Reachability Analysis. IEEE Transactions on Robotics. 2014;30(4):903-918.

[138] Li L, Huang W, Liu Y, et al. Intelligence Testing for Autonomous Vehicles: A New Approach. IEEE Transactions on Intelligent Vehicles. 2016;1(2):158-166.

[139] Schultz A, Grefenstette J, De Jong K. Adaptive testing of controllers for autonomous vehicles. In: Proceedings of the 1992 Symposium on Autonomous Underwater Vehicle Technology; ????

[140] Lutz A, Schick B, Holzmann H, et al. Simulation methods supporting homologation of Electronic Stability Control in vehicle variants. Vehicle System Dynamics. 2017;55(10):1432-1497. Available from: https://doi.org/10.1080/00423114 . 2017.1322705 .

[141] Kutluay E, Winner H. Validation of vehicle dynamics simulation models - a review. Vehicle System Dynamics. 2014 2;52(2):186-200. Available from: https : //doi.org/10.1080/00423114.2013.868500.

[142] Hoesli S, Klomp M, Bleicher H. Virtual Validation of Steering Systems in Passenger Vehicles. ATZ worldwide. 2018;120(12):46-51. Available from: https: //doi.org/10.1007/s38311-018-0173-x.

[143] Google Self-Driving Car Project Monthly Report; 2016. 